\title{
SST and ADITYA Tokamak Research in India
}

\author{
Dhiraj Bora, ADITYA Team \& SST-1 Team, \\ Institute for Plasma Research, \\ Bhat, Gandhinagar-382 428, INDIA
}

Received on 3 July, 2001

\begin{abstract}
Steady state operation of tokamaks plays an important role in high temperature magnetically confined plasma research. Steady state Superconducting Tokamak (SST) programme in India deals with the development of various technologies in this direction. SST-1 [1] machine has been engineered and is being fabricated at the Institute for Plasma Research. The objectives of the machine are to study physics of plasma processes under steady state condition and develop the technologies related to steadystate operation. Various sub-systems are being prototyped and developed. SST-1 is a large aspect ratio machine with a major radius of $1.1 \mathrm{~m}$ and a plasma minor radius of $0.2 \mathrm{~m}$ with elongation of 1.7 to 1.9 and triangularity of 0.5 to 0.7 . It has been designed for 1000 sec operation at $3 \mathrm{~T}$ toroidal magnetic field. Neutral beam Injection and Radio frequency heating systems are being developed to heat the plasma. Lower hybrid Current Drive system would sustain $200 \mathrm{kA}$ of plasma current during $1000 \mathrm{sec}$ operation. ADITYA tokamak [2] has been upgraded with new diagnostics and RF heating systems. Thomson Scattering and ECE diagnostics have been operated. 200 kW Ion Cyclotron Resonance Heating (ICRH) and $200 \mathrm{~kW}$ Electron Cyclotron Resonance Heating $(\mathrm{ECRH})$ systems have been successfully commissioned. RF assisted initial breakdown experiments have been initiated with these systems.
\end{abstract}

\section{Introduction}

Tokamak research in India has matured over the years. After experiements on pulsed ohmic plasmas in ADITYA Tokamak, experiments on auxiliary heating, current drive and other areas of research with high power radio frequency $(\mathrm{RF})$ and microwave sources have begun. At the same time, a major programme is under way to study steady state operation of tokamaks. With this aim in mind a steady state superconducting Tokamak (SST-1) has been designed and is being built at Institute for Plasma Research, India. This paper describes the present status of ADITYA Tokamak research and SST-1 project.

\section{SST - 1 Tokamak}

The basic objectives of the SST programme are :

1. To run SST-1 Tokamak discharges for $1000 \mathrm{sec}$ and to investigate physics issues related to heat removal and particle control at the boundaries.

2. To develop the technologies required to achieve the above goal.

Many of the conventional issues studied in Tokamak physics will be addressed during operation in steady state scenario. Particle and energy confinement during noninductive current drive in steady state operation will be studied. Effect of impurity confinement and ELMS on energy confinement in steady state will be studied. Studies on stability limits, disruptions, thermal instability and vertical displacement events (VDE) in Plasma with noninductive current drive will be emphasised. Study of different divertor configurations will form an important area of research in the SST-1 programme. Some experiments in advanced tokamak regimes would also be attempted.

\section{Machine features}

SST-1 has a major radious $(\mathrm{R})$ of $1.1 \mathrm{~m}$ and a minor radius of $0.2 \mathrm{~m}$, a toroidal field of $3.0 \mathrm{~T}$ at the plasma centre and a plasma current of $220 \mathrm{KA}$. The proposed plasma will have an elongation $(\kappa)$ of $1.7-1.9$ and triangularity $(\delta)$ in the range of $0.4-0.7$. Initially hydrogen plasma discharges would be produced for a maximum duration of 1000 Sec. Ohmic driven plasma current would be replaced by lower hybrid wave driven noninductive current in the plasma to sustain the discharge for 1000 Sec. Fig. 1 shows the comparison of the cross sectional view of ADITYA tokamak and SST-1. 


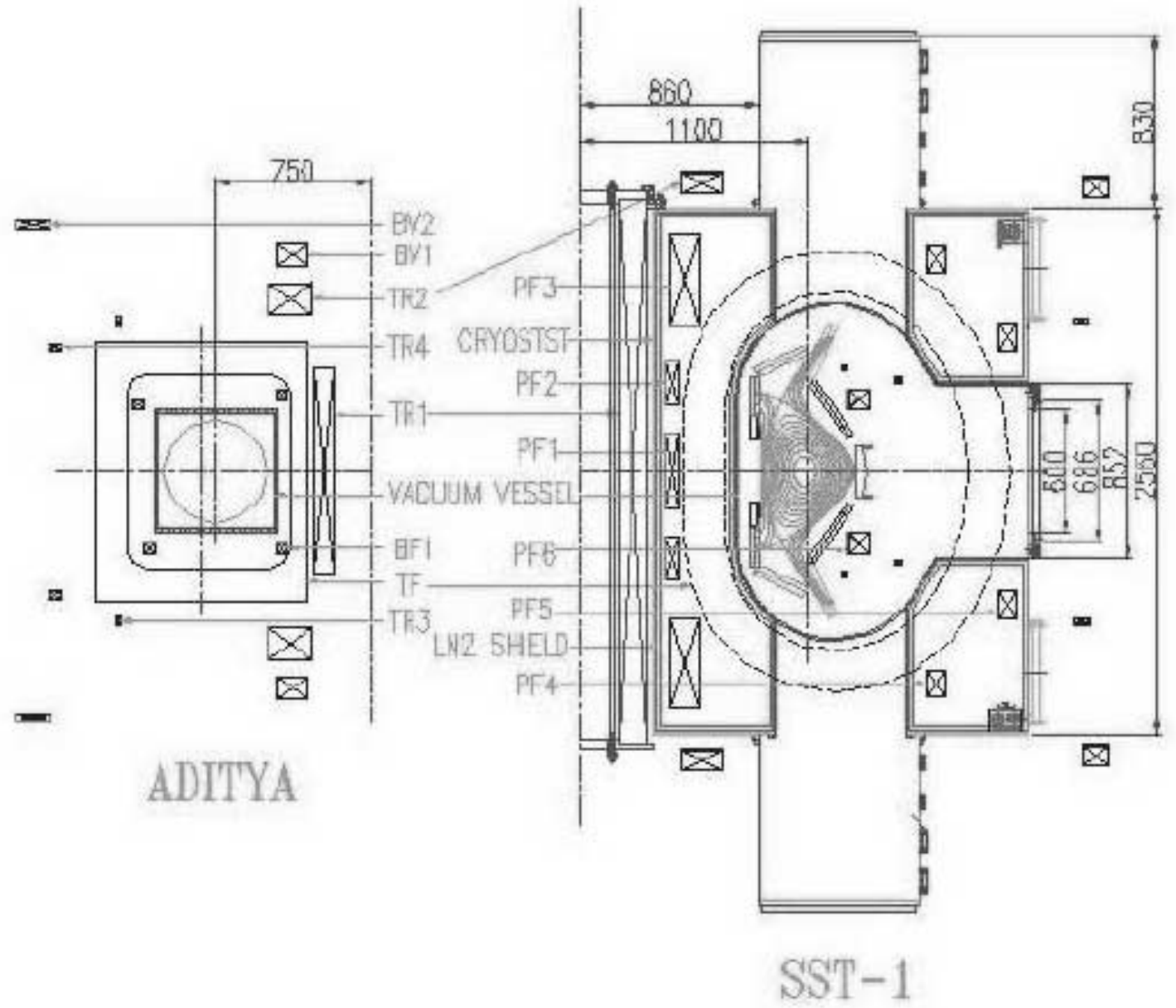

Figure 1. Cross sectional view of ADITYA Tokamak and SST-1.

SST - 1 Machine would operate with superconducting toroidal and poloidal field coils. Discharge would be initiated with the help of an ohmic transformer using copper coils giving a volt-seconds capability of $\sim 1.4 \mathrm{~V}$ s. An ultra high vacuum compatible vessel would house the plasma facing components. A high vacuum cryostat would enclose all the SC coils and the vacuum vessel. To reduce the heat load on $\mathrm{SC}$ coils Liquid Nitrogen $\left(\mathrm{LN}_{2}\right)$ cooled thermal shields would be used between the vacuum vessel and the cryostat.

\section{Magnet system}

The magnet system comprises of TF coil system, PF coil system, ohmic transformer, vertical field coils and vertical postion control coils. Position of various magnets in relation to plasma is shown in Fig. 1.

\section{Toroidal field (TF) coil system}

The toroidal field coil system will produce a $3 \mathrm{~T}$ magnetic field on the plasma axis with $<2 \%$ ripple within the plasma volume. The TF assembly has been designed to operate in steady state and withstand plasma disruptions and VDEs without quenching. The whole assembly will be cooled down and warmed up within 15 days. TF coil system consists of 16 modified D shaped TF coils arranged geometrically around the major axis. These superconducting coils are wound from specially designed NbTi / $\mathrm{Cu}$ CICC manufactured by Hitachi Ltd., Japan. A total of 17.28 MA T at a peak current of $10 \mathrm{KA}$ per turn will produce a field of $3.0 \mathrm{~T}$ at the plasma centre and a maximum of $5.1 \mathrm{~T}$ at the TF conductor. Each TF coil is made of six double 'pancakes' and each pancake having nine turns. Inter-double pancake joints are conventional soldered joints. Cryogenic grade E glass tapes with half overlap are used for turn to turn insulation in pancakes. NEMA grade G-11 CR sheets separate each of the pancakes. Six double pancakes with a ground wrapping are vacuum pressure 
impregnated with epoxy and then shrunk fitted into a stainless steel (SS316L) casing which supports most of the electromagnetic load.

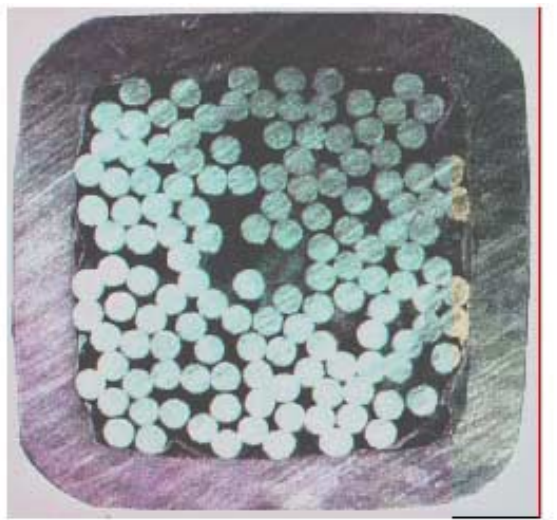

Figure 2. A cross sectional view of SST-1 CICC with 135 strands in it.

The CICC is made up of 135 strands cabled in a 3 $\times 3 \times 3 \times 5$ cabling pattern and conduited in a SS304L conduit (Fig. 2).

It is designed for an operational current of $10 \mathrm{KA}$ at $5 \mathrm{~T}$ and $4.5 \mathrm{~K}$, with a critical current of $36 \mathrm{KA}$. The nominal critical current for each strand is $272 \mathrm{~A}$ at $5 \mathrm{~T}$ and $4.5 \mathrm{~K}$. The qualification tests have been carried out on strands at $5 \mathrm{~T}$ and $4.2 \mathrm{~K}$. Various tests have been performed on the strands. Dependence of strand critical current on temperature for various fields is shown in Fig. 3. The results indicate that degradation in the strands due to cabling and jacketing is less than $5 \%$ and a critical current of $\geq 35 \mathrm{kA}$ at $5 \mathrm{~T}, 4.2 \mathrm{~K}$ is ensured. Test conducted at Kurchatov Institute at Moscow on a model coil fabricated out of $600 \mathrm{~m}$ long strand [3] indicate stability of the conductor for TF coils of SST1. Further tests have been performed to establish the limit on the ramp rates for various current levels in the conductor.

All the TF coils are connected in series and are protected against quenching by a suitable dump resistor, switching and sensing system. Typical dump time constant is $12 \mathrm{sec}$ and the maximum voltage across the coils at the dump time is $\pm 600 \mathrm{~V}$ with respect to ground.

The TF coils are further supported on a base support system consisting of a ring with 16 caintilevered beams. The ring rests on eight columns that are inside the cryostat and have LN2 intercepts to minimize the conduction loss at $4.5 \mathrm{~K}$ as the cold mass load is transferred from these columns to the main machine support structure. The main machine support is comprised of 8 columns, supporting the base frame of the cryostat and the cold mass. They are firmly grouted to ground.

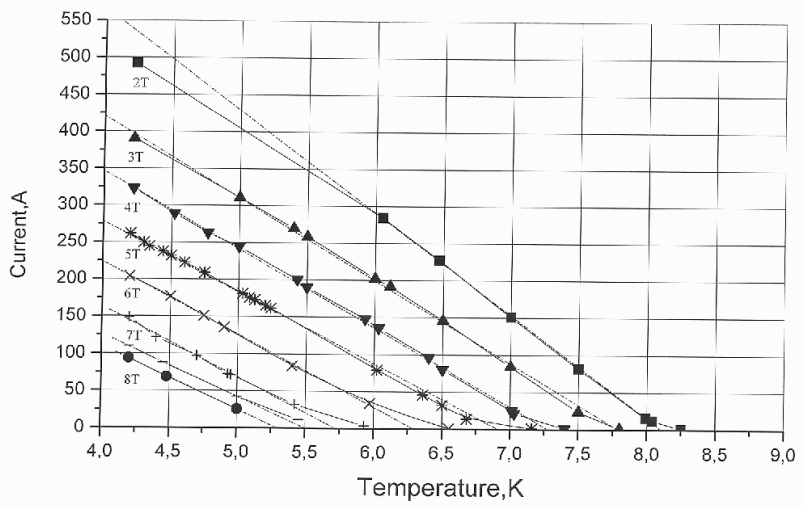

Figure 3. Strand Critical Current vs Temperature for various field.

\section{Poloidal field (PF) coil system}

The design and optimization of the PF coil system has been done based on a free boundary, axisymmetric, ideal MHD equilibrium model. The PF Coil system is comprised of nine SC coils (PF1 to PF5) and two normal copper coils (PF6). The PF6 coils are placed in the bore of the TF coils inside the vaccum vessel. They are required to obtain plasma shapes with high triangularity. In the absence of PF6 coils, large number of ampere turns are required in the external $\mathrm{PF}$ coils to obtain similar triangularity. The PF coils allow for a wide range of elongation and triangularity and support wide range of plasma equilibria. Design goals include various startup scenario, feasibility of similar operation during plasma current rampup, double and single null operation.

The designed PF system allows for plasma elongation in the range $1.7-1.9$, triangularity in the range $0.4-0.7$, plasma inductance in the range $0.75-1.4$ and poloidal $\beta$ in the range $0.01-0.85$. A slot divertor configuration is also accommodated in the design.

\section{Ohmic transformer and Vertical field coils}

An ohmic transformer, comprised of a central solenoid (TR1) and two pairs of compensated coils (TR2 and TR3) are used for plasma startup and initial current rampup. The SC poloidal coils are not being used as they demand that the rate of change of currents in these coils be limited below a certain level to avoid quenching of these coils during startup phase. Therefore to establish an equilibrium field on a fast time scale, the ohmic transformer is made out of hollow copper conductors that has a storage capacity of $1.4 \mathrm{~V} \mathrm{~s}$ and would be used for producing circular plasma with $100 \mathrm{KA}$ current for 1 second duration. A pair of vertical field coils will keep the plasma in equilibrium during the initial phase. 
The current drive will then be taken over by LHCD and the PF coils will be ramped up at a slow rate, typically in $3 \mathrm{~s}$ in a pre-programmed fashion to achieve the desired plasma equilibrium. It will also provide the divertor configuration for an elongated triangular plasma.

\section{Feedback control coils}

It is well known that vertically elongated plasma is inherently unstable to perturbations in the vertical direction. To arrest the growth rate of this instability, a set of passive stabilizers are placed inside the vacuum vessel. This would reduce the growth rate to $\sim 38 \mathrm{Sec}^{-1}$ corresponding to the most unstable equilibrium for $\kappa=$ 1.9. Further stabilisation is provided by active feedback using a pair of coils placed inside the vacuum vessel.

These coils have a major radius of $1.35 \mathrm{~m}$ and are connected in a saddle configuration. They are displaced by $\pm 0.5 \mathrm{~m}$ from the equatorial plane. During long pulse operation, minor radial shifts of the plasma will also be taken care of by these coils.

\section{Cryogenic system for SST - 1}

The SC magnets will be cooled using forced flow of supercritical helium (SHe) through void space in the CICC. A total flow $\sim 0.3 \mathrm{Kg} / \mathrm{s}$ is required to keep the SC temperature well below the current sharing temperature in the presence of peak pulsed loads. Vapour cooled current leads connect the magnets to power supplies. He at the cold end evaporates to gas Helium at $\sim 300 \mathrm{~K}$ at the warm end of the lead. A Helium refrigerator / liquefier with cold circulation system for $\mathrm{SHe}$ is being set up for this purpose. $\mathrm{LN}_{2}$ shields are provided between cold mass at $4.5 \mathrm{~K}$ and warmer surfaces to minimize the heat loads on magnets and support system at $4.5 \mathrm{~K}$. A LN $\mathrm{LN}_{2}$ storage and distribution system is provided for this purpose.

Heat loads experienced by SC coils include steady state heat loads and losses during operation. A total of $180 \mathrm{~W}$ is estimated as the steady state heat load. During a plasma pulse, SC coils are subjected to a pulsed load of $\sim 125$ KJ. Such pulses would be repeated after each $5000 \mathrm{sec}$. for a maximum of six times during a day. Such a load would evaporate $\sim 150 \mathrm{~L} / \mathrm{hr}$ of LHe to helium gas at $\sim 300 \mathrm{~K}$ in the current leads of the $\mathrm{SC}$ coils.

A closed cycle LHe plant is being installed to cater for both the steady state and the transient heat loads. The plant has a refrigeration capacity of $650 \mathrm{~W}$ at 4.5 $\mathrm{K}$, and $200 \mathrm{~L} / \mathrm{hr}$ liquefaction at a pressure of $1.2 \mathrm{bar}$ which includes the provision of $\sim 250 \mathrm{~W}$ refrigeration capacity for the heat dissipation in the cold circulation pump. This capacity is achieved with LN2 precooling. It is possible to operate the plant without precooling to reduced capacity.

$\mathrm{LN}_{2}$ cooled radiation shields are provided between the $\mathrm{SC}$ coils and the vaccum vessel and between the coils and the cryostat. Under different operating and conditioning phases the $\mathrm{LN}_{2}$ consumption would vary. A maximum of $\sim 1500 \mathrm{~L} / \mathrm{hr}$ consumption is anticipated for all the systems. Therefore commercially available $\mathrm{LN}_{2}$ would be stored in tanks for distribution. Tanks have been procured and installed. Various components of the distribution line are under fabrication.

The helium plant has been procured from M/s. Air Liquide cryogenie, France. It is under process of installation.

\section{Vaccum vessel, cryostat and pumping system}

The vacuum vessel is being fabricated in sixteen modules which would be welded in situ. Each module consists of a vessel sector, an interconnecting ring and three ports. The vessel sector is placed between two TF coils while the ring sector is located in the bore of the TF coil. There are totally 32 vertical ports and 16 radial ports. It is an ultra high vacuum system with partial pressures, for all gases, less than $1 \times 10^{-8}$ torr except hydrogen. The SS304L vacuum vessel has a height of $1.62 \mathrm{~m}$, a midplane width of $1.07 \mathrm{~m}$, a total volume of $16 \mathrm{~m}^{3}$ and a surface area of $75 \mathrm{~m}^{2}$. The vessel would be baked to $525 \mathrm{~K}$. Other wall conditioning techniques such as discharge cleaning, boronization etc. are also envisaged.

The cryostat is a sixteen sided high vacuum chamber also made of SS $304 \mathrm{~L}$. A base pressure of $1 \times 10^{-5}$ Torr would be maintained in the cryostat. It has a volume of $35 \mathrm{~m}^{3}$ and surface area of $59 \mathrm{~m}^{2}$.

$126 \mathrm{~m}^{2}$ surface area of $\mathrm{LN}_{2}$ cooled panels are placed between all surfaces at temperatures higher than $85 \mathrm{~K}$ and surfaces at $4.5 \mathrm{~K}$. Panels are formed of $8 \mathrm{~mm}$ diameter tubes vacuum brazed to $1 \mathrm{~mm}$ thick SS $304 \mathrm{~L}$ sheets.

During normal operation 10,000 L/S pumping speed is provided to achieve $1 \times 10^{-8}$ torr base pressure. Similar speed is also provided for the cryostat. Two turbomolecular pumps, each with $5000 \mathrm{~L} / \mathrm{S}$ speed, will be used for the purpose. Main gas load anticipated from the Vessel is during steady state operation. For this purpose, sixteen turbomolecular pumps each with 5000 $\mathrm{L} / \mathrm{S}$ speed at $10^{-3}$ torr for hydrogen will be connected to sixteen pumping lines on the vacuum vessel. 


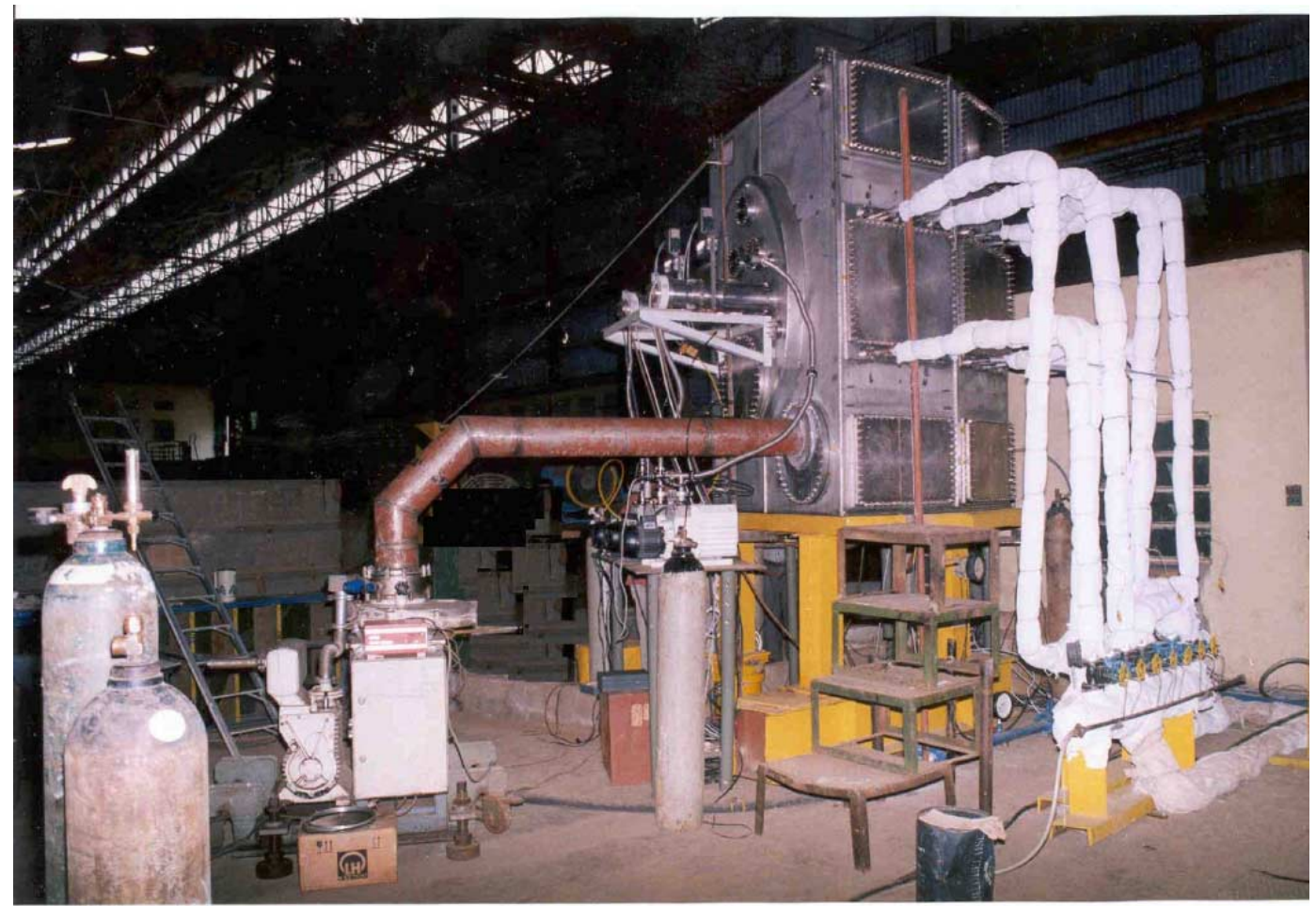

Figure 4. A view of the SST-1 proto-type being tested in laboratory.

The vacuum vessel and the cryostat will be pumped down from atmospheric pressure to $10^{-3}$ torr using two separate Roots pumps of $2000 \mathrm{~m}^{3} / \mathrm{h}$ capacity.

A prototype of one vessel sector with ports, two ring sectors and one eighth section of the cryostat has been fabricated and tested successfully at $\mathrm{M} / \mathrm{s}$. Bharat Heavy Electricals Limited, Tiruchirapalli, India, which has established all the processes for final fabrication. The prototype has been shifted to site and it has been commissioned in the vacuum laboratory. Fig .4 shows the proto-type system under testing. In the meantime fabrication of the main system is well underway.

Central radial port of the vacuum system along with top and bottom port of the cryostat are seen in the picture. All add on systems will be tested for functional compatibility with the system on this proto-type.

\section{Plasma facing components (PFC)}

Divertors and baffles, poloidal limiters and passive stabilisers are the constituents of the PFC of SST - 1 [4]. Heat and particle removal during steady state operation is the prime concern during the design of PFC. Inboard and outboard divertor plates are designed for the worst possible heat loads by assuming different inout and up-down asymmetry for the divertors. The normal incident heat flux at the inboard and ouboard strike points is 1.6 and $5.6 \mathrm{MW} / \mathrm{m}^{2}$, respectively. The poloidal inclination of the outboard divertor plates is adjusted so as to have an average heat flux at the strike points of less than the allowed limit of $0.6 \mathrm{MW} / \mathrm{m}^{2}$. The target points of the inboard and the outboard divertor plates have been chosen at a distance as large as possible from the null point. This reduces the electron temperature at the target plate and decreases the impurity flux from the divertor region. A closed divertor configuration is achieved by incorporating a baffle that helps to increase the neutral pressure in the divertor region and improves neutral particle recycling and reduces electron temperature. This geometry also helps in efficient pumping of the divertor region. The slot width has been designed by using neutral particle code DEGAS and the results have been confirmed by the use of the Plasma and neutral transport coupled code B2 - EIRENE.

One of the main objectives of SST - 1 is to achieve steady state particle removal from the vessel during steady state discharges with a steady state plasma density of $1 \times 10^{19} \mathrm{~m}^{-3}$, confinement time of $12 \mathrm{~ms}$. The particle exhaust expected from the core is 22 torr $\mathrm{l} / \mathrm{s}$ with the divertor region pressure at $1 \times 10^{-3}$ torr. The pumping speed required for the divertor region is $11,000 \mathrm{l} / \mathrm{s}$. With all the geometrical factors taken into account, wide operating range of plasma density and 
uncertainty of particle confinement time, the divertor pumping system has been designed to provide a pumping speed of $63,000 \mathrm{l} / \mathrm{s}$ at $1 \times 10^{-3}$ torr pressure of hydrogen gas at pumping port of the vacuum vessel.

A pair of poloidal movable limiters is provided to assist plasma breakdown, current rampup and current rampdown, and to protect $\mathrm{RF}$ antennae and other invessel components during normal operation, VDEs and during disruptions. The inboard and outboard limiters are designed to handle about $4 \%$ of the input power during steady state operation. A safety limiter is positioned $50 \mathrm{~mm}$ away from the seperatrix on the inboard side. Plasma facing surface of the outboard limiter is designed with appropriate poloidal curvature to avoid interference during divertor operation. The limiter surfaces are toroidally profiled to reduce the heat flux.

Passive stabilizers of conducting structures surrounding the plasma are provided to reduce the growth rate of the vertical stability and make active feedback control possible. Two pairs of stabilisers, one on the inboard and one on the outboard side of the plasma are placed above and below the midplane. To allow plasma startup with an ohmic flux swing, an electric break is incroporated. The top and bottom stabilizers are connected in the saddle configuration with a current bridge at the location of this break. They are designed to handle heat fluxes at $0.25 \mathrm{MW} / \mathrm{m}^{2}$.

The plasma facing surfaces of stabilizer plates are covered with $20 \mathrm{~mm}$ thick graphite tiles. Active cooling is provided to maintain their temperature below $150^{\circ} \mathrm{C}$. Isostatically pressed fine grain graphite is chosen as the baseline armour material for the PFC of SST1. Copper base alloys such as copper-zirconium and copper-chromium zirconium are selected as the substrate material for divertor and limiter assemblies due to their good thermal conductivity, good mechanical behaviour at elevated temperatures for long durations and high electrical conductivity at elevated temperatures. The PFCs are actively cooled to keep surface temperature less than $1000^{\circ} \mathrm{C}$. They are bakable up to $350^{\circ} \mathrm{C}$.

Poloidal limiter system is being fabricated and is in advance stage of completion.

\section{SST-1 Plasma shape extraction}

Due to very long pulse length (maximum of 1000 seconds) SST-1 control system would need to execute dynamic control of discharges to achieve the objectives of the discharge. One of the first requirements for this is the need to develop means by which information available from the discharge is processed in real time (of the order of about 1-2 ms) to allow controller to take real time decisions about the discharge progress. Artificial Neural Network technique has been utilized to develop algorithms to extract SST-1 plasma shape boundary from magnetic measurements in real time. Algorithms have also been developed for prediction of major plasma disruption and for the prediction of density limit boundary. These have been tested with ADITYA discharge data and found to give satisfactory performance.

A multi-layered feed-forward neural network (NN) is used to extract the tokamak plasma parameters from external magnetic measurements. It is also used to optimize the number and locations of the magnetic diagnostics designed for the tokamak. The magnetic measurements will be utilized to achieve real-time control of plasma position, shape and some global profiles. An NN with 93 inputs (comprising 23 flux loop differences, 24 pairs of normal and tangential magnetic probes and 22 Mirnov coils), 25 nodes in a single hidden layer and 17 outputs (the physical parameters of the plasma) is trained and tested, and the results are compared with the traditional statistical method of function parametrization (FP). Both techniques appeared well suited for the purpose, but a definite improvement with $\mathrm{NN}$ is observed. Although simulated measurements are used in this study, confidence regarding the network performance with actual experimental data is ensured by testing the network's noise tolerance with Gaussian noise of up to $10 \%$. Finally, three possible methods of ranking the diagnostics in decreasing order of their importance are suggested, and the $\mathrm{NN}$ is used to optimize the magnetic sensors designed for SST-1. The results from the three methods are compared with one another and also with FP. Magnetic probes within the plasma facing side of the outboard limiter are ranked high. FP and one of the NN methods showed a distinct tendency to favour the probes located in the remote regions of the vacuum vessel, proving the importance of redundancy. Fault tolerance of the optimized network is tested. The results obtained should, in the long run, help in the decision regarding the final effective set of magnetic diagnostics to be used in SST-1 for rapid recovery of plasma control parameters.

\section{Current drive and auxiliary heating}

Radio Frequency (RF) [5] and Neutral Beam Injection (NBI) [6] methods are planned in SST-1 for noninductive current drive and plasma heating. Two main factors have been considered during the development of these auxiliary systems, namely, high heat flux (1 $\mathrm{MW} / \mathrm{m}^{2}$ ) incident on the plasma facing antennae components and fast feedback for constant power input due to small energy confinement time ( $\sim 12 \mathrm{msec})$. Auxiliary heating systems would also be used for other 
experiments. RF break down and wall conditioning have proved very beneficial in tokamak operation. It is specially useful for wall conditioning between long plasma shots in machines with SC toroidal field coils where the field is not ramped down after each shot. At high densities, fast wave current drive in the core of the plasma proves advantageous than lower hybrid current drive. Electron cyclotron current drive is also useful for profile shaping. These considerations have been taken into account during design of the rf systems for SST-1.

\section{Lower hybrid current drive (LHCD) sys- tem}

LHCD system would be used as the prime method of steady state plasma current drive in a circular as well as an elongated plasma. The system has been optimised for efficient current drive for $1.5 \mathrm{~T}$ and 3.0 $\mathrm{T}$ operation, for different plasma elongation and triangularity, and for plasma inductance value of 0.75 to 1.4. Lower hybrid waves with asymmetric spectrum $\left(\mathrm{N}_{\|} \sim 1.8-4.0\right)$ would be launched by changing the phase between adjacent waveguides from 40 to 160 degrees using high power phase shifters through a radial port at $3.7 \mathrm{GHz}$ to drive plasma current during different operating scenarios. Computational analysis using LSC and WDFP codes show that $220 \mathrm{kA}$ plasma current would be driven with available power of 1.1 MW for circular plasma at $2 \times 10^{13} \mathrm{~cm}^{-3}$ average density and $3 \mathrm{~T}$ toroidal magnetic field. For the same machine and plasma parameters and plasma current, $860 \mathrm{~kW}$ of power is sufficient if the plasma is heated by auxiliary ICRH power of $650 \mathrm{~kW}$. Since the launcher is made of two rows of narrow waveguides, they could be fed with equal power and independently phased at different angles so as to radiate different spectra. Under similar conditions it is possible to drive the same plasma current at a total input power of $600 \mathrm{~kW}$. Current driven for a shaped plasma is being estimated with the help of ACCOME code. $1 \mathrm{MW}$ of $\mathrm{CW}$ power would be supplied from two klystrons to feed a grill consisting of 64 narrow waveguides in two rows placed on the equatorial plane of LFS radial port.

The main components of the system are low power section, high power amplifier (klystrons), high power transmission line, high power phase shifter (to vary the launched spectrum), high power directional coupler (to monitor forward and reflected power), vacuum window and grill. Each Thomson CSF klystron (TH2103D) operates at $3.7 \mathrm{GHz}$ and delivers $250 \mathrm{~kW}$ each in two arms of WR284 waveguide for $1000 \mathrm{sec}$. The power level in each of the 64 channel is around $15 \mathrm{~kW}$ and is definitely easy to handle. Finally the power will be coupled to the plasma through the grill interfaced by a vacuum window assembly. Four dummy waveguides of quarter wavelength are at the four corners to reduce fringe field effect. A protection limiter is placed around the grill to protect the launcher from direct particle load. It is proposed that the grill would be positioned at $1.3 \lambda_{n}$ ( or equivalently at $3.25 \lambda_{Q}$ behind the LCFS). Due to the heat load on the tiles and under constant cooling the temperatutre rise of the tiles are envisaged to be about $\sim 310$ degree.

Zr-Cr copper, with high thermal conductivity and high tensile strength has been chosen for the fabrication of the grill to withstand the thermal stresses developed due to the above discussed heat loads. Calculation shows that the estimated peak temperature rise of the copper grill is $\sim 116^{\circ} \mathrm{C}$ producing a thermal stress of $\sim 220 \mathrm{MPa}$ which is below the yield stress for $\mathrm{Zr}-\mathrm{Cr}$ copper. Finite element ANSYS analysis confirms the analytic calculations. Disruption stress analysis shows that a maximum of $\sim 13 \mathrm{kN}$ force will be generated on the grill. The calculation is based on a simple model which assumes that $220 \mathrm{kA}$ plasma current disrupts within $0.1 \mathrm{msec}$ ( decay rate $\sim 2.2 \mathrm{GA} / \mathrm{sec}$ ). Similar results are obtained with SPARK code ( Eddy current) analysis.

The vacuum window which accommodates 64 narrow waveguides would be made out of 99.9Titanium alloy to avoid thermal cracks and microcracks due to differential thermal expansion. Other transmission line components like $\mathrm{E} / \mathrm{H}$ bend, transformer, narrow waveguide have been prototyped, tested for high power and are being fabricated.

In the meantime two klystrons (TH2103D) have been successfully installed and commissioned on dummy loads [7]. A test system has been established around one of these klystrons to test high power components. Intense klystron conditioning by applying high voltage to the electrodes has been conducted in the absence of input rf power. Gradually the pulse length and the applied voltage is increased. RF input is applied once the tubes are conditioned. Beam current is monitored and the output microwave power is measured with the help of directional couplers. Maximum of 200 $\mathrm{kW}$ output is measured for a voltage of $-53 \mathrm{KV}$ and a beam current of $\sim 9 \mathrm{~A}$. Operation is limited due to the current capacity of 10 A for the HVDC power supply. Fig. 5 shows the output power and beam current as a function of cathode voltage. Typical $1000 \mathrm{sec}, \sim 200$ $\mathrm{KW}$ microwave power output is depicted in Fig 5 . 


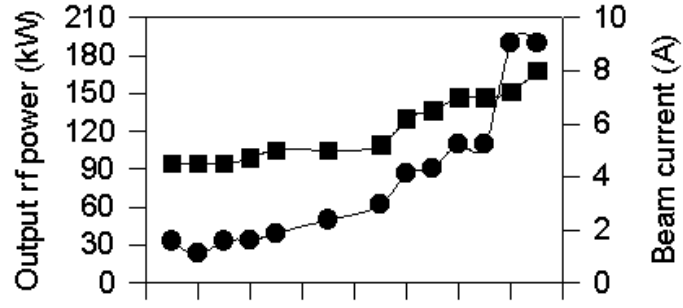

$38 \quad 40 \quad 42 \quad 44 \quad 46 \quad 48 \quad 5052 \quad 54$

Cathode voltage $(\mathrm{kV})$

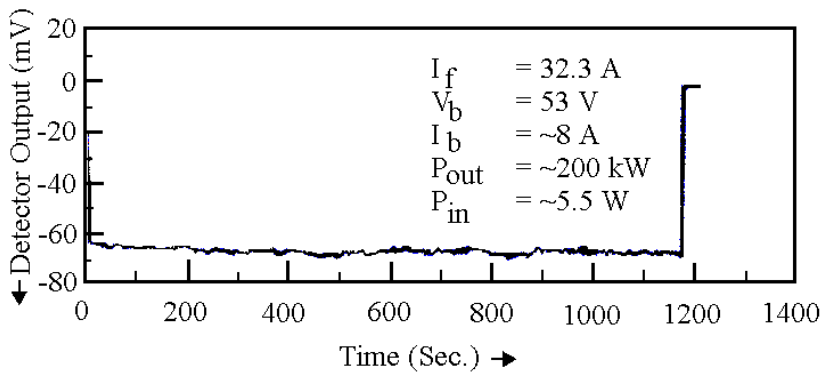

Figure 5. Figure on the left shows output power and beam current as a function of cathode voltage whereas figure on the right shows detector output corresponding to $200 \mathrm{~kW} \mathrm{rf}$ power for $1000 \mathrm{~s}$.

\section{Ion cyclotron resonance heating (ICRH) system}

Ion cyclotron heating is chosen to heat the plasma to $1.0 \mathrm{KeV}$, during pulse length of $1000 \mathrm{~s}$. A $1.5 \mathrm{MW}$ ICRF system would operate at different frequencies between $20-92 \mathrm{MHz}$ for different heating scenarios at 1.5 $\mathrm{T}$ and at $3.0 \mathrm{~T}$ operation.

Conventional design criteria have been implemented in developing the $1.5 \mathrm{MW}, 1000 \mathrm{~s}$ RF system. Various efficient heating scenarios (e.g. 2nd harmonic of majority species at $1.5 \mathrm{~T}$ and $3.0 \mathrm{~T}$, minority $\mathrm{D}$ in $\mathrm{H}$-plasma) would be implemented. The generator is tetrode based and modular. For the last high power stage, tetrode has been selected which has wider frequency bandwidth. RF generator will be placed in the generator room about $90 \mathrm{~m}$ away.

A $90 \mathrm{~m}$ long pressurised 9", $50 \mathrm{ohm}$ transmission line would feed the antennae. RF power is divided by $3 \mathrm{~dB}$ hybrid coupler and then by two Tees to obtain equal power at each antenna. The entire transmission line will be pressurized at 3 bar to avoid breakdown. Water flow through inner conductor has been selected as the most optimized method after prototype testing. Slow stub matching (in secs) and automatic matching with VVC (20-30 msec) as well as fast frequency ( $\sim 2 \mathrm{~ms})$ matching techniques would be used to optimise match- ing during the discharge. Reflected power would be replenished by increasing the RF input power as per the feedback signal till slow matching is achieved. Interface would be made of SS304L for better mechanical strength and coated with copper $(\sim 100 \mu)$ for better rf transmission. For $1000 \mathrm{~s}$ operation interface will be actively cooled (full length of inner conductor and a narrow portion of outer conductor). Antennae are designed using two powerful codes BRACC and SWHAP. Four antennae (each carrying $375 \mathrm{~kW}$ of power), placed on low field side (out of neutral beam sight) at $3.5 \lambda_{q}$ behind the last closed surface, will heat plasma to 1 $\mathrm{KeV}$ during $1000 \mathrm{~s}$. Design goal for power density is $1.2 \mathrm{~kW} / \mathrm{cm}^{2}$. Antenna is shielded from the plasma by 30 no. of Faraday shields in a single column. Antenna assembly is made of SS304L. Graphite tiles will form the protection limiters around the antenna box. Expected heat load on the antenna assembly is $80 \mathrm{~W} / \mathrm{cm}^{2}$. Maximum temperature at the graphite tiles would be $211^{\circ} \mathrm{C}$. Thermal analysis has been conducted with the help of ANSYS for all the plasma facing components. Maximum thermal stress on the antenna and on protection tiles are $330 \mathrm{MPa}$ and $25 \mathrm{MPa}$ respectively. Disruption stress analysis shows that a maximum of $1.4 \mathrm{kNm}$ of torque will be generated on the antenna structure. Impurities generated due to physical sputtering of shield material limits the power density. Analytical calculation shows that acceleration of ions in the rf sheath does not produce significant sputtered SS ions.

The system is in advanced stage of fabrication. 1MW water dummy load is erected and undergoing commission at $200 \mathrm{~kW}$ level of RF power.

\section{Electron cyclotron resonance heating (ECRH) system}

ECRF system at $200 \mathrm{~kW}, 82.6 \mathrm{GHz}$ will be used initially to preionize and startup of SST-1 discharges. A Gyrotron source capable of delivering $200 \mathrm{~kW} \mathrm{CW}$ has been ordered. Second harmonic ' $\mathrm{X}$ ' and 'O' mode launching would be used during $1.5 \mathrm{~T}$ operation. Provision for low as well as high field side launch has been made. The output mode of the gyrotron with internal mode converter is $\mathrm{HE}_{11}$. A transmission line consisting of d.c. break, bellows, mitre bend, polariser and corrugated waveguide terminating with a barrier window will be used to transmit power from the gyrotron to the tokamak. The total attenuation of the line is 1.1 dB. Quasi optical reflecting mirror system has been designed to steer the microwave gaussian beam toroidally. Low Field Side launcher consists of two reflectors: one focusing mirror and a plane mirror. For long pulse operation the reflectors require effective cooling. A separate mirror box is attached to the radial port flange 
to accomodate the reflectors along with their cooling arrangement. Similarly in the top launch scheme, two mirrors (one focusing and one plane) are used to focus the beam. The distance between focusing reflector and baffle is $1150 \mathrm{~mm}$. A $25 \mathrm{~mm}$ aperture is provided in the baffle for beam entrance. Radius of the beam waist at the baffle is selected to be $18 \mathrm{~mm}$. $14.5 \%$ power is lost at the baffle. The temperature rise of the smallest reflector is around $350{ }^{\circ} \mathrm{C}$, taking $1 \%$ absorption of microwave power on the reflector for 1000 second operation. Negligible part of EM power is absorbed during $\mathrm{O}$-mode launch in its first pass. After reflection from the vessel wall the beam is absorbed in the centre. The beam waist radius at the wall is $29 \mathrm{~mm}$ and corresponding rise in temperature of wall for $1 \%$ absorption is approximately $700{ }^{\circ} \mathrm{C}$. During plasma disruption the EM forces experienced by the box, and its support is $2 \mathrm{KN}$. Force on the plane reflector due to toroidal field component is $\sim 20 \mathrm{~N} / \mathrm{m}$ while the force on the support and reflector due to $\mathrm{B}_{R}$ component is $\sim 40 \mathrm{~N} / \mathrm{m}$. The force on the plane reflector due to toroidal field component $\sim 120 \mathrm{KN}$.

The gyrotron and the transmission line is in advanced state of fabrication at the contractor's place. All other subsystems are being fabricated inhouse after prototype testing.

\section{Neutral beam injection (NBI) system}

The power required in NBI in the low and high density phases for SST-1 machine is 0.5 and $1.7 \mathrm{MW}$, respectively. This requirement will be fulfilled by tangential injection of the beam, corresponding to maximum absorption at $0.98 \mathrm{~m}$ radius of tangency in the plasma. The beam parameters and their effect on the plasma have been modeled using the 1-D transport code BALDUR. Hydrogen or Deuterium beam will be used at 30 - $80 \mathrm{keV}$. The injection is expected to raise the ion temperature to $\sim 1 \mathrm{keV}$, contribute to a fuelling of $\sim 4$ torr $\mathrm{L} / \mathrm{s}$, impart a toroidal momentum of $\sim 100 \mathrm{~km} / \mathrm{s}$, and drive a current of $\sim 40 \mathrm{kA}$ at the core of the plasma. The power will be delivered from a single beam line and the dynamic range of voltage will be accommodated in a single source.

Engineering designs have been completed for the ion source, neutralizers I and II, deflection magnet, Vtarget, cryogenic conversion system, $\mathrm{LN}_{2}$ distribution system, water supply system and vacuum vessel for the injector systems. The tender documents for almost all the components have been forwarded to the industry for their technical proposal.

The ion source component level design has already been carried out successfully. There have also been simulation studies carried out on the magnetic fields in order to find out the optimal magnetic geometry on the back plate for a maximum field free region on the back plate. The neutralizer has been designed to dissipate a total power of $250 \mathrm{KW}$ in the two stages. The system is designed to handle a gas throughput of $\sim 50 \mathrm{~T} \mathrm{l} / \mathrm{s}$ for providing an integrated line density of $\sim 0.35$ Torr $\mathrm{Cm}$. An active magnetic shielding is provided on the second stage neutraliser to reduce the stray magnetic fields inside the neutraliser to $<1 \mathrm{G}$.

Detailed computations using 2D/3D magnet modeling computer codes and analytical particle trajectory calculations, using the field distributions obtained from the above mentioned codes, have led to the finalization of a large ion deflection magnet system. The major design goal has been to get the best possible field uniformity and field distributions in the 3 directions ( $\mathrm{z}$ axis being the direction of propagation of the beam) for a magnet having the least possible pole gap to pole depth ratio. It has been found that for a ratio of $1: 2(30 \mathrm{~cm} / 60 \mathrm{~cm})$, the obtained field uniformities are of the order of $1 \%$ in the directions prependicular to the beam direction and $\sim 6 \%$ along the beam direction. Analytical trajectory calculations indicate power densities of less than $1 \mathrm{~kW} / \mathrm{cm}^{2}$ on the dumps placed at an angle of $4^{\circ}$ with respect to the vertical. The calculations will be benchmarked by developing a prototype magnet having $1 / 6^{t h}$ dimensions as those for the main magnet. Vendor finalization has been concluded for the fabrication of the magnet.

V-target consists of two arms, one having an assembly of 10 and the other one an assembly of 11 HTE's. The design allows for an angular movement of $7.2^{\circ}$ about hinge points of each arm. Links mechanism is used for opening and closing both the arms simultaneously. To transfer motion from outside to inside of vacuum, a bellow is used. The whole structure is supported on a support platform, which is adjustable in height, by $30 \mathrm{~mm}$.

Engineering of the $\mathrm{LN}_{2}$ and LHe distribution system has also been carried out. The $\mathrm{LN}_{2}$ distribution system is based on a simple scheme of supplying the required flow from a phase separator maintained at a working pressure of 1.8 bar. The phase separator is filled by a $320 \mathrm{l} / \mathrm{h}$ line branched from the main line. The distribution system for $\mathrm{LN}_{2}$ is presently under fabrication. A $4.5 \mathrm{~K}$ to $3.8 \mathrm{~K}$ LHe conversion system supplies the liquid Helium through a sub-atmospheric distribution system to the NBI cryopumps. The conversion system is based on the simple concept of pumping on the liquid Helium for lowering the saturation temperatures. The conversion system sources the liquid Helium from the SCMS refrigerator. 


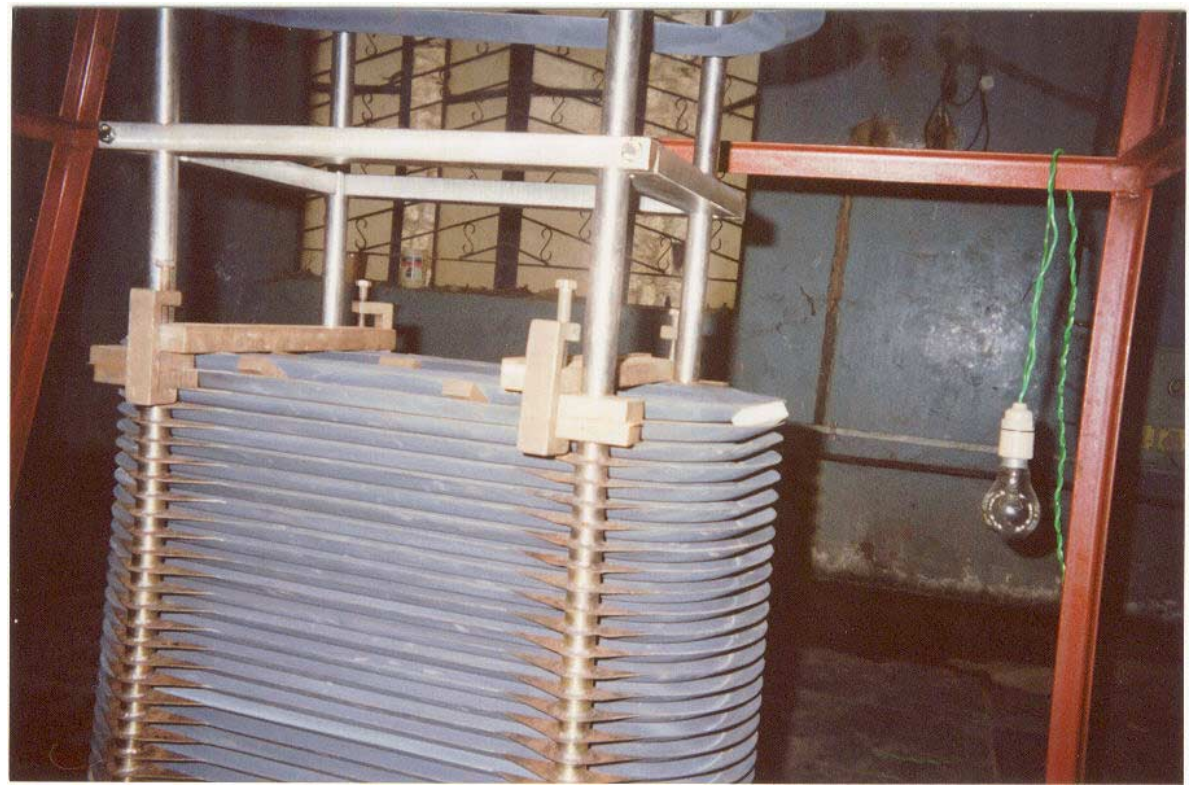

Figure 6. Cryo-pump full scale fabrication.

The fabrication of one module of a $10^{5} \mathrm{l} / \mathrm{s}$ cryo condensation pump has been completed. The pump thus manufactured is now ready for test of its engineered performance.

Special considerations have been made while deciding on the engineering design of the duct, which is a critical system for long pulse beam injection. Modularity has been incorporated in the design by the incorporation of standard heat transfer elements to protect from the power flux expected due to a re-ionization loss of $\sim 3$ $\%$. A critical element in the duct is the torus duct isolation system which incorporates a $1 \mathrm{~m}$ diameter bellow and a ceramic isolator rated for $\sim 5 \mathrm{kV}$.

\section{Prototype development of power supplies}

One of the major achievements has been the development of a prototype of a regulated high voltage power supply (RHVPS). A $490 \mathrm{~kW}(14 \mathrm{kV}, 35 \mathrm{~A})$ RHVPS has been successfully tested. This has also led to the validity of several components like multi-secondary (20) transformers, IGBT based switched power supplies, controller that initiates and regulates the functioning of the RHVPS with a fast dynamic response time, a menu driven control console with provision for variable rise and fall times, low voltage regulation and a low ripple without any filter.

Development of a unique low voltage simulator will serve as a test bed for a number of model tests to be carried out before implementation. The development has now paved the way for the development of a $80 \mathrm{kV}$, 8 MW RHVPS required for the auxiliary heating systems.

Prototypes of the Arc power supply (AC/DC converters) and Filament Power Supply (AC/AC converters) have also been developed at the vendors site. The salient features of the Arc Power Supply include low current ripple (low loss L-C filter design), use of PID loop with a low drift for the load and line regulation and IGBT switches at final stage for fast turn ON/OFF times. For the filament power supply, the salient features include a special synchronisation circuit for obtaining a phase shift of $<10$ between all 8 inverters, user friendly input and output control commands and a Variable Voltage Constant Frequency inverter for minimising the size of the filament transformer at $400 \mathrm{~Hz}$ constant frequency operation.

\section{Prototype development of heat transfer elements}

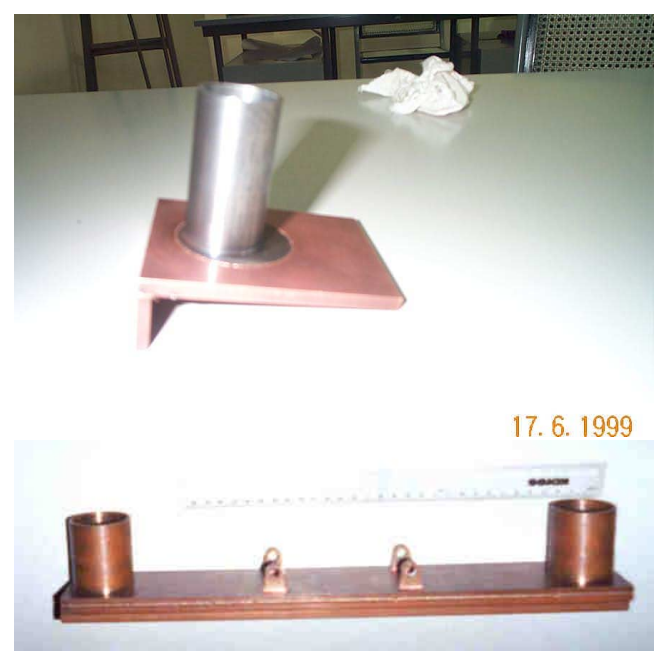

Figure 7. Heat transfer element - prototype fabrication. 
2D finite element analysis related to thermal and structural properties lead to the design of the heat transfer elements using the $\mathrm{Cu}-\mathrm{Cr}-\mathrm{Zr}$ alloy. The elements are designed to dissipate a heat load of $15 \mathrm{MW} / \mathrm{m}^{2}$. Repeated trials on the material and the jointing process (EB Welding) have now led to the successful development of one module. This has undergone tests for thermal cycling. In the meantime the test stand in the form of a large vacuum system has been fabricated and tested. The same is now being erected and tested at site.

\section{Diagnostics for SST - 1}

Various diagnostics will be used in SST - 1. Parameters like plasma current, position, shape, $\beta_{p}$, density, electron and ion temperatures in the core, edge and divertor regions, impurity concentrations, radiated power, q profile, surface temperatures of various PFC, fluctuations in the basic parameters over wide range of frequency will be measured. Long pulse operations have implications on the diagnostics techniques.

Other diagnostics include FIR interferometer, Thomson scattering, ECE, charge exchange, thermography, soft and hard x-ray monitoring, visible and VUV spectroscopy and motional stark effect (MSE) diagnostics. The MSE diagnostics will utilize the NBI system of SST -1 .

\section{Thomson scattering system}

For the temporal evolution of the electron temperature $\left(\mathrm{T}_{e}\right)$ and density $\left(\mathrm{n}_{e}\right)$ profiles over $1000 \mathrm{sec}$ SST-1 plasma, a multi-point, multi-pulse Thomson scattering diagnostics system has been designed. In order to have a better understanding of the plasma parameters, it has been decided to probe the different regions with spatial and temporal resolutions as given in table. For achieving the above task three such Thomson scattering systems have been designed for SST-1, namely :

1. Vertical Imaging System (VIS)

2. Horizontal tangential Imaging System (HTIS)

3. Divertor Imaging System (DIS)

These three systems will together cover a parameter range of, $\mathrm{n}_{e} \geq 1 \times 10^{12} \mathrm{~cm}^{-3}$ and $\mathrm{T}_{e}: 20 \mathrm{eV}$ to 3.5 $\mathrm{keV}$ over the 1000 second plasma duration.

\begin{tabular}{|c|c|c|c|c|c|}
\hline $\begin{array}{l}\text { Plasma } \\
\text { region }\end{array}$ & $\begin{array}{l}\text { Spatial } \\
\text { coverage }\end{array}$ & Time resolution & $\begin{array}{l}\text { Spatial } \\
\text { resolution }\end{array}$ & $\begin{array}{l}\text { Spatial } \\
\text { points }\end{array}$ & $\begin{array}{l}\text { Measurement } \\
\text { durations }\end{array}$ \\
\hline $\begin{array}{l}\text { Core } \\
\text { (vertical } \\
\text { profile) }\end{array}$ & $\begin{array}{l}\text { Partial } \\
(\mathrm{Z}=-24 \\
\text { to }+24 \mathrm{~cm})\end{array}$ & $\begin{array}{l}-33 \mathrm{msec} \\
5.5 \mathrm{msec}-\text { Burst } \\
\text { mode with } \sim 1 \mathrm{msec} .\end{array}$ & $1 \mathrm{~cm}$ & 19 & 1000 seconds \\
\hline $\begin{array}{l}\text { Core and } \\
\text { Edge } \\
\text { (Midplane } \\
\text { tangential } \\
\text { profile) }\end{array}$ & $\begin{array}{l}\text { Full (from } \\
\text { inner to } \\
\text { outer } \\
\text { separatrix) }\end{array}$ & $\begin{array}{l}33 \mathrm{msec} \\
5.5 \mathrm{msec} \\
\text { Burst mode with } \\
\sim 1 \mathrm{msec} .\end{array}$ & $\begin{array}{l}1 \mathrm{~cm} \text { at core } \\
\text { and } 0.5 \mathrm{~cm} \text { at } \\
\text { at edge }\end{array}$ & 19 & $1000 \mathrm{sec}$. \\
\hline $\begin{array}{l}\text { Divertor } \\
\text { region }\end{array}$ & $\begin{array}{l}\text { Full (from } \\
\mathrm{X} \text {-point to } \\
\text { the plate }\end{array}$ & $\begin{array}{l}33 \mathrm{msec} \\
5.5 \mathrm{msec} . \\
\text { Burst mode } \\
\text { with } \sim 1 \mathrm{msec} .\end{array}$ & $1 \mathrm{~cm}$ & 10 & $1000 \mathrm{sec}$. \\
\hline
\end{tabular}

Table 1: Table showing the spatial and temporal resolution for Thomson scattering system

The systems are in advance state of procurement and fabrication.

\section{Zeeman polarimetry}

This diagnostic involves the measurement of the poloidal field distribution that would yield the plasma current density profile. This information is essential for understanding confinement, stability and energy balance of tokamak plasmas.

To extract the magnetic field information from the line profiles, which are dominated by the thermal effect, the circular polarization associated with the spectral line, which is proportional to the component of magnetic field along the direction of observation is measured. The absolute strength of the magnetic field is obtained from the difference in intensities between the right and left circularly polarized components when observed along the magnetic field direction.

Intensity calculations for the intensity profiles for HeII(Aditya) and C-VI(SST-I) have been successfully completed. Based on it the conceptual design for the Zeeman Polarimeter has been finalized. As soon as the 
engineering design is completed, experiment for the HeII line on Aditya would be performed before installing the same on SST-I machine.

\section{Spectroscopy diagnostics}

The spectroscopy diagnostics for SST-1, during the initial operational phase will consist of the following: A $0.3 \mathrm{~m}$ grazing incidence spectrograph with 3 interchangeable toroidal gratings $(15-170 \mathrm{~nm}$, resolution $0.4 \mathrm{~nm}, 10-110 \mathrm{~nm}$, resolution $0.3 \mathrm{~nm}$, and 10-30 nm , resolution $<0.1 \mathrm{~nm}$ ). This spectrograph will be UHV coupled to the tokamak and view a central radial chord. It will be equipped with an ICCD detector capable of recording the full spectral range (mentioned above) every 10 millisec.

A 1 meter multi-track visible spectrograph equipped with a CCD detector achieving a resolution of $\sim 0.05$ nm over $300-800 \mathrm{~nm}$ will be used. 8 optical fibers will be used to transport light from various regions of the tokamak to the entrance slit of the spectrograph simultaneously. Spectra of width $15 \mathrm{~nm}$ centered around any selected wavelength, from each of the 8 tracks can be recorded every 150 millisec. By limiting to one track, spectra can be recorded every 10 millisec indefinitely, or a "burst" of 8 spectra can be recorded within about 10 milliseconds, which can be repeated about every 150 millsec.

A plasma imaging system will be set up to view the cross section of the torus almost tangentially through one of the radial view ports. A coherent fiber optic bundle consisting of 10 micron fibers will carry a reduced image of the plasma to a 25 frames-per second CCD camera. The spatial resolution at the object plane is expected to be about $2.5 \mathrm{~mm}$.

A similar set up for in-situ illumination and inspection of the inside of the tokamak is also being envisaged.

For continuous monitoring of a number of selected spectral lines of Hydrogen, and low ionization states of $\mathrm{O}$, $\mathrm{C}$, etc. , so as to estimate the fluxes of these species from various plasma facing surfaces, filter based PMT detectors will be used. About 12 such channels having a temporal resolution of $\sim 1$ millisec and 5 channels of $\sim 10$ microsec resolution, are planned. The latter ones will be utilized for the detection of fast plasma events like VDE's, Elms, L-H transition etc.

The various optical components and electronics needed for the above diagnostics are being identified, procured and assembled. The issues related to data acquisition at high rates and for long duration are being worked out.

\section{IR Thermography}

This diagnostic involves the measurement of heat fluxes on divertors and limiters. These measurements are necessary in order to determine the material requirement for next generation devices and to understand the physics of Scrape Off Layer (SOL). IR imaging of these target plates provide quantitative information about both the steady state and transient heat fluxes to the target plates.

Order has been placed for the IR camera needed for the experiment. Intensity calculations have been performed for all the five viewing objects in order to determine the $\mathrm{S} / \mathrm{N}$ ratios for them. The optical design for all the target plates is completed and the engineering design is in progress.

\section{Electron cyclotron emission diagnostics}

The objectives of ECE diagnostic measurements are: (1) Electron temperature measurement (2) Energy confinement study which needs electron temperature profile (3) Core plasma stability study which needs electron temperature time evolution with fast time scale $(10 \mu \mathrm{s})$ (4) Electron thermal energy transport study which needs time evolution of electron temperature at different radii simultaneously and (5) study of non thermal electron distribution parameters.

There are advantages and disadvantages in various methods of measurement. However, the first four objectives can be achieved by radiometer measurement with many advantages to its credit compared to other techniques. And the last objective need continuous and complete ECE spectrum which can only be achieved by Michelson interferometer.

\section{SST-1 Radiometer}

In SST-1, toroidal field will remain 1.5 or 3 Tesla during different phases of operation. Since, the toroidal field will vary from 1.1 to 3 Tesla, three radiometers will be used for SST-1 ECE measurement to determine $\mathrm{T}_{e}$ profile for any value of the field within this range: (1) An available ADITYA radiometer having a frequency range $60-88 \mathrm{GHz}$ will be utilized for field range of 1.1 to $1.6 \mathrm{~T}$ operation stages of SST-1.

(2) Intermediate Frequency (IF) section of $1-40 \mathrm{GHz}$ has been developed.

For the first phase, a radiometer of frequency 91 - 130 $\mathrm{GHz}$ with IF frequency ranging from $1-40 \mathrm{GHz}$ has been developed. It has central toroidal field coverage of 1.7 to 2.3 Tesla for half or more than half plasma cross section for $\mathrm{T}_{e}$ measurements.

In the second phase, radiometer at $131-170 \mathrm{GHz}$ will be developed. It has central toroidal field coverage of 2.3 to 3 Tesla for half or more than half plasma cross 
section, for $\mathrm{T}_{e}$ measurements.

(3) A Michelson interferometer having a frequency range $70-700 \mathrm{GHz}$ is required to be developed to study the non-thermal electron distribution function parameters.

The engineering design of the F-Band and D-Band radiometers has been completed. The procurement of the F-Band and IF section components is being made.

\section{Microwave interferometer}

A single channel $136 \mathrm{GHz}$ interferometer is designed to measure the chord averaged density of plasma in SST-1. Engineering design has been completed. The procurement of all the active and passive components will be completed during the year.

\section{Microwave reflectrometer}

A broad band reflectometer (O-mode) to measure the density profile in SST-1 has been designed. A microwave source (Gunn Oscillator), whose output can be swept from 10-20 GHz, is used. The planned $\mathrm{O}$ - mode propagation allows determination of density profile for the range from $1 \times 10^{12}$ to $5 \times 10^{12} \mathrm{~cm}^{-3}$. The spatial and temporal resolutions are about $1 \mathrm{~cm}$ and $1 \mu \mathrm{sec}$, respectively. Engineering design has been completed.

Most of the diagnostics are being fabricated tested as per engineering design.

\section{Data acquisition and control system}

SST-1 Data Acquisition Group is engaged in designing a High speed lossless data acquisition system for diagnostics of SST-1.

In pursuance of continuous data acquisition needs of various diagnostics PXI based data acquisition system of NI has been chosen The system supports a back plain activity at $132 \mathrm{Mbytes} / \mathrm{sec}$ with various trigger line and clock for synchronizations. The MXI III controller offers fiber optic connectivity between host and controller supporting data transfer rate of $1 \mathrm{Gbits} / \mathrm{sec}$. The system supports multiple chasis and a maximum distance of 200 meters.

SST - 1 control system [8] is based on distributed control system for efficient management. The control system has been divided into machine control system, discharge control system and diagnostics supervisory control. Various technical subsystems will be configured and operate with the help of the machine control system with a hierarchial control structure. The low level, front end, systems would be based on field bus devices, programmable logic controllers and VME systems.

A control area network is selected as the default standard for the field bus.

The discharge control system will be activated during the discharge. All discharge related subsystems will be controlled by it. Real time control systems would be based on VME standard for real time digital data communication. The discharge control system will take decisions regarding operational phases and control the real time system. The diagnostics supervisory control will advise diagnostics control regarding expected plasma parameters and expected diagnostics setting depending on the planned experiment. It will also collect some data from diagnostics for plasma state analysis purposes.

\section{Operational scenario of SST-1:}

Two phases of operation are envisaged for SST-1. First phase will involve various steps to finally reach at $1000 \mathrm{sec}$ steady state operation at 3T toroidal field. Second phase then will involve advance tokamak operations. 


\begin{tabular}{|c|c|c|c|c|c|c|c|}
\hline \multirow{4}{*}{$\begin{array}{l}\text { Basic Plasma } \\
\text { parameter }\end{array}$} & \multicolumn{4}{|c|}{ "Phase I (L mode) } & \multicolumn{3}{|c|}{ Phase II (H mode) } \\
\hline & (Step 1) & (Step 2) & (Step 3) & (Step 4) & Low & Low & High \\
\hline & Circular & Circular & Elongated & Elongated & Low & Low & High \\
\hline & Ohmic & LHCD & $\begin{array}{l}\text { LHCD } \\
(0.5 \mathrm{MW})\end{array}$ & $\begin{array}{l}\text { LHCD } \\
(1.0 \mathrm{MW})\end{array}$ & power & field & power \\
\hline $\mathrm{B}_{T}(\mathrm{~T})$ & 1.5 & 1.5 & 1.5 & 3 & 3 & 1.6 & 3 \\
\hline $\mathrm{I}_{p}(\mathrm{kA})$ & 110 & 110 & 150 & 220 & 220 & 100 & 220 \\
\hline $\mathrm{q}^{*}($ cylindrical $\mathrm{q})$ & 2.5 & 2.5 & 4.1 & 5.7 & 5.7 & 6.6 & 5.7 \\
\hline $\mathrm{H}$ factor, & - & 1 & 1 & 1 & 2 & 2 & 2 \\
\hline$\tau_{E} / \tau_{I T E R 89-P}$ & & & & & & & \\
\hline$<n>\left(\times 10^{19} \mathrm{~m}^{-3}\right)$ & 3 & 0.5 & 0.8 & 1 & 2 & 2 & 5 \\
\hline$<n_{o}>\left(\times 10^{19} \mathrm{~m}^{-3}\right)$ & 4.5 & 0.75 & 1.2 & 1.5 & 3 & 3 & 7.5 \\
\hline $\mathrm{P}_{a u x}(\mathrm{MW})$ & - & 0.17 & 5 & 1 & 1 & 1 & 5 \\
\hline $\mathrm{Z}_{e f f}$ & 1.7 & 5 & 5 & 1.7 & 1.7 & 1.7 & 1.7 \\
\hline Elongation $\left(\kappa_{x}\right)$ & 1 & 1 & 1.8 & 1.8 & 1.8 & 1.8 & 1.8 \\
\hline Triangularity $\left(\delta_{x}\right)$ & 0 & 0 & 0.66 & 0.66 & 0.66 & 0.66 & 0.66 \\
\hline Elongation $\left(\kappa_{95}\right)$ & 1 & 1 & 1.6 & 1.6 & 1.6 & 1.6 & 1.6 \\
\hline Triangularity $\left(\delta_{95}\right)$ & 0 & 0 & 0.54 & 0.54 & 0.54 & 0.54 & 0.54 \\
\hline$\tau_{E}(\mathrm{~ms})$ & 12 & 10 & 11 & 12 & 26 & 12 & 13 \\
\hline$<T_{e}>(\mathrm{keV})$ & 0.24 & 0.82 & 1 & 1.8 & 2 & 0.9 & 1.9 \\
\hline$<T_{i}>(\mathrm{keV})$ & 0.09 & 0.32 & 0.4 & 0.74 & 0.8 & 0.36 & 0.78 \\
\hline$<T_{e} o>(\mathrm{keV})$ & 0.39 & 1.37 & 1.7 & 3.1 & 3.3 & 1.5 & 3.2 \\
\hline$<T_{i} o>(\mathrm{keV})$ & 0.16 & 0.55 & 0.67 & 1.2 & 1.3 & 0.6 & 1.3 \\
\hline$\beta(\%)$ & 0.18 & 0.1 & 0.2 & 0.12 & 0.25 & 0.39 & 0.61 \\
\hline$\beta_{N}(\%, \mathrm{mT} / \mathrm{MA})$ & 0.48 & 0.28 & 0.4 & 0.32 & 0.68 & 1.3 & 1.7 \\
\hline$\beta_{p}(\%)$ & 0.33 & 0.19 & 0.46 & 0.49 & 1.1 & 2.3 & 2.6 \\
\hline $\mathrm{f}_{b s}$ & 0.08 & 0.04 & 0.1 & 0.12 & 0.25 & 0.55 & 0.62 \\
\hline $\begin{array}{l}\text { BS collisionality } \\
\text { correction }\end{array}$ & 0.57 & 0.11 & 0.12 & 0.07 & 0.09 & 0.2 & 0.14 \\
\hline
\end{tabular}

Table 2: Table showing operating parameter scenerio for SST - 1

Table 2 shows typical operating parameters in various steps of phase I and Phase II. Plasma operation will commence with circular, pulsed plasma driven by ohmic field for a period of $\sim 1$ sec. This current will be sustained in the second step with the help of lower hybrid wave. About $200 \mathrm{KW}$ power will be used in this step. The toroidal field will be $1.5 \mathrm{~T}$ during these steps. Step three involves divertor operation at the same toroidal magnetic field. In the presence of a circular LHCD driven plasma, the poloidal field coils would be brought in a slow time scale of $3-4$ sec to produce elongated divertor plasma. In the final step of Phase I, the toroidal magnetic field will be raised to designed $3 \mathrm{~T}$ and long steady state plasma discharges would be obtained. Slow switching of the poloidal coils has been simulated and equilibrium is ensured. Full design parameters would then be attempted and a total of 1MW of auxiliary power would be introduced. Advance tokomak configurations will be explored in phase II of SST-1 operation. In order to improve $\beta$ value with the same auxiliary power, one would oper- ate at a lower toroidal field of $1.6 \mathrm{~T}$. Further improvement can be obtained in $\beta_{P}$ and $\beta_{N}$ by operating at lower currents. Experimentations with VH mode, nonmonotonic q profiles and significant bootstrap current will be tried. However, these could be attempted at the suitable modifications of PFCs for higher power handling capabilities and enhancement of RF power systems.

\section{ADITYA Tokamak}

ADITYA, a medium size Tokamak, is being operated for over a decade. It has a major radius of $0.75 \mathrm{~m}$ and minor radius of the plasma is $0.25 \mathrm{~m}$. A maximum of $1.2 \mathrm{~T}$ toroidal magnetic field is generated with the help of 20 toroidal field coils spaced symmetrically in the toroidal direction. The major subsystems and parameters of the machine have been described earlier [9]. ADITYA is regularly being operated with the transformer-converter power system. $\sim 100 \mathrm{msec} 80$ $100 \mathrm{kA}$ plasma discharges at toroidal field of $8.0 \mathrm{kG}$ are being regularly studied. During this period experiments 
on edge plasma fluctuations, turbulence and other related works have been conducted. Standard diagnostics have been employed during these measurements. Fig. 8 gives a view of ADITYA with the auxiliary heating systems attached to it.

ADITYA has been upgaded. Vacuum system has been upgraded in terms of more cleaning facilities. Some more diagnostics have been integrated and made on-line. Some are in the design/fabrication phase. To increase the plasma energy content during the discharge, auxiliary heating systems have been integrated. A 20 - 40 MHz, 200 KW Ion Cyclotron Resonance Heating (ICRH) system has been integrated to ADITYA vacuum vessel. A $28 \mathrm{GHz}, 200 \mathrm{KW}$ gyrotron based electron cyclotron resonance heating (ECRH) system has been successfully commissioned on ADITYA tokamak. Some neural network analysis to predict disruptions and density limit on ADITYA have also been performed.

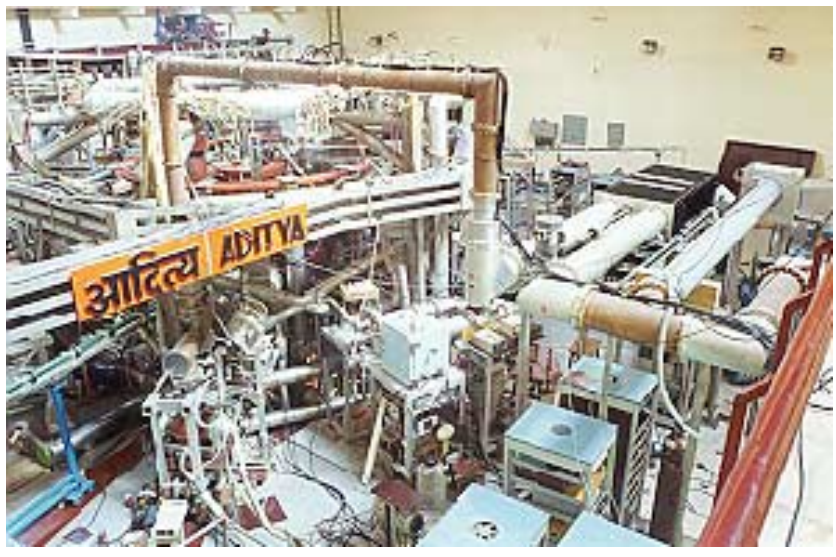

Figure 8. Present View of ADITYA Tokamak.

\section{Vacuum System and Wall Conditioning}

It has been realised over the years that clean wall condition plays a very important role in obtaining reproducible discharges. Therefore, the main objective has been to maintain good vacuum and to perform wall conditioning to reduce impurity level. A series of experiments have been carried out to understand behaviour of quadrupole mass analyser in tokamak ADITYA.

Wall cleaning techniques for ADITYA include automated Glow Discharge Cleaning (GDC) for a maximum period of 12 hours, Pulse Discharge Cleaning (PDC), Electron Cyclotron Resonance (ECR) discharge cleaning. ECR and/or PDC discharge cleaning is continued for few hours to 24 hours as and when required.

Other types of coating, viz. Boronization and Lithiumization have been proposed. Lithium coating is a new technique for wall conditioning that is being regularly used in other machines. Initial experiments for lithium coating in laboratory have been carried out successfully. The effect of lithium coating on the wall condition has been observed in tokamaks. Though still in the phase of development, the potential advantage of these techniques is their application in real time during the discharge, making them particularly suitable for steady state devices viz. SST-1.

An experiment in tokamak ADITYA with in-situ lithium conditioning to study effect of lithium conditioning on Aditya Discharges has been performed. The aim of lithium conditioning was to reduce hydrogen recycling, impurity influx and to improve plasma parameters viz. plasma density, temperature, confinement time and MHD activities. Lithium conditioning has been done by the evaporation of lithium in Aditya vacuum vessel during discharge cleaning. Partial pressures of lithium and oxygen are monitored with Quadrupole Mass Analyser. An increase in partial pressure of lithium (100\%) has been observed whereas for oxygen, it is reduced to $50 \%$. The density is 1 $\times 10^{13} \mathrm{~cm}^{-3}$ with a flat top for 30 millisecond. At $78 \mathrm{KA}$ of plasma current, sawteeth was expected but not observed. Presence of lithium is also monitored by spectroscopy. An increase in $\mathrm{H}$-alpha (instead of reduction) and reduction in Hard $\mathrm{X}$ rays were observed. The plasma parameters thus were improved. Plasma current for most of the discharges was more than 97 KA for 100 millisecond.

Experiments to study lithium coating on vacuum vessel wall are being continued. Solid lithium target probes have been prepared. These probes are introduced in a UHV system and lithium coating experiments have been performed successfully. More experiments would be performed to study the effect of lithium coating. Lithium coating on limiter is also proposed in ADITYA.

\section{Gas Feed System}

The gas feed line and related systems for feeding of pure hydrogen gas to the vacuum system have been modified. A series of experiments to study impurity level for different conditions of the gas feed system have been conducted. Fast gas puffing during plasma discharge has been carried out successfully.

Efficient gas fuelling is a prime requirement of tokamaks. In ADITYA, after obtaining good ultimate vacuum in the system, hydrogen gas is introduced for plasma production. During the discharge, pressure in the system is maintained at required value. There are different methods for gas fueling. The major draw backs of the normal gas puffing system are the spread 
in velocity profile as angular distribution of incoming particle, and the slow velocity component of gas particles that can not penetrate into the plasma centre which would ultimately cool the plasma edge. A considerable number of rundown particles are adsorbed on the wall surfaces and limiters, which are released from the surfaces during the discharge, become an uncontrollable factor in density control. A new method of gas fuelling in tokamaks with molecular beam injection has been developed. Its efficiency is $50 \%$. The beam can travel inside plasma for few centimeters thereby reducing edge cooling. MBI also reduces recycling of the fuel gas.

A molecular beam injection system for tokamak ADITYA with a Piezo-electric gas inlet valve modified inhouse to inject molecular beam has been developed. This valve is mounted on a vacuum system in the laboratory to verify its functions with various input gas pressures. It is observed that as the input gas pressure increases, the pressure in the centre of the vessel increases. But the pressure remains low near the vessel wall.

\section{Limiter bias experiment}

An understanding of the mechanism of improved particle/energy confinement in a tokamak is of paramount importance for the design and operation of a fusion reactor. Several experiments have been done for this purpose, viz., biased probe, biased limiter, edge cooling, preferential heating of ions at the edge etc. Recently we have carried out a limiter bias experiment on ADITYA. The ADITYA limiter consists of 16 segments of graphite tiles and thus provides an opportunity to study the role of biasing with a set of biasing in different configurations.

Results of a preliminary experiment of limiter biasing on ADITYA are reported .One of the limiter tiles is biased up to $-500 \mathrm{~V}$ with respect to the vessel. When the limiter current drawn exceeds 300 A various diagnostic signals indicate an improvement of particle/energy confinement. $\mathrm{H}_{\alpha}$ radiation as well as floating potential fluctuation (monitored by Langmuir probes) show significant reduction. The amplitude of floating potential reduced by $80 \%$ at all frequencies. Before biasing the limiter frequency spectrum of the fluctuation shows a peak at $10-15 \mathrm{KHz}$, which disappeares during biasing. Signals from the central detector of the soft X-ray camera indicate a rise in temperature by $50-100 \mathrm{eV}$ within 3-4 ms of the application of the bias. A sharp decrease up to $50 \%$ in $\mathrm{H}_{\alpha}$ radiation has been observed.

\section{Diagnostic systems:}

New diagnostic systems include Thomson scattering, Lithium beam, ECE, Bolometer, Soft X-ray etc. Polarimetry is being designed and fabricated to be tested on ADITYA and finally used in SST-1. Some of these systems are described below.

\section{Thomson scattering system}

The aim is to provide electron temperature and density measurements of ADITYA plasma on shot to shot basis. The system is capable to measure electron temperature $\left(\mathrm{T}_{e}=20 \mathrm{eV}-500 \mathrm{eV}\right)$ and electron density $\left(\mathrm{n}_{e}=5.0 \times 10^{12}-10^{13} \mathrm{~cm}^{-3}\right)$ profiles of ADITYA plasma (from $\mathrm{z}=+22 \mathrm{~cm}$. to $-14 \mathrm{~cm}$ ) with a spatial resolution of $1 \mathrm{~cm}$ and temporal resolution of $50 \mathrm{nsec}$. The system is based on a Ruby Laser (10J, $20 \mathrm{nsec}$ ) that enters the plasma through an extended bottom vertical port and exits through a top vertical port.

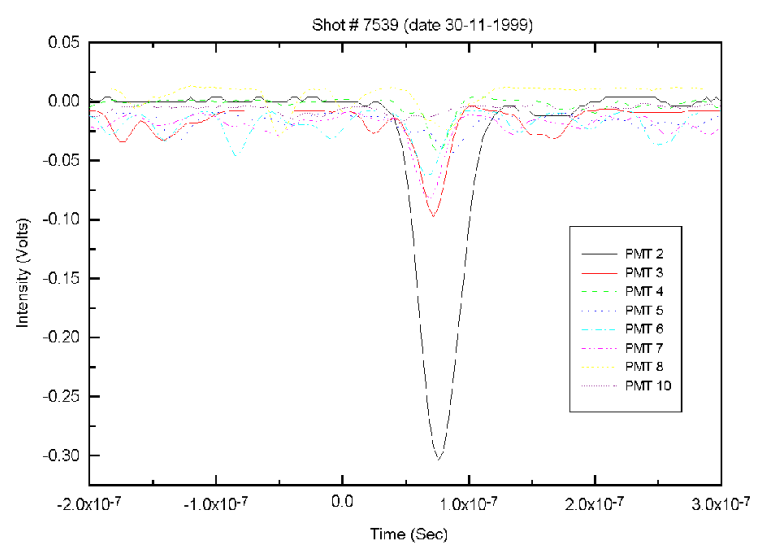

Figure 9. ADIYTA Thomson scattering Signal in presence of plasma.

The $90^{\circ}$ scattered photons are collected through a radial port using a f/5 optical system ( a set of different lens systems) collected scattered spectrum is dispersed using a grating spectrometer (1200 grooves $/ \mathrm{mm}$, resolution $8 \mathrm{~A}^{\circ}$, focal length $1 \mathrm{~m}$ ) and exit of spectrometer is coupled with a fiber bundle to detectors ( 10 channels, PMT of RCA make).

The output signals from PMT are amplified and digitized using CAMAC modules and the data is acquired and analyzed using a PC located at the laser control room.

Wavelength and spectral calibrations of spectrometer, PMTs are done regularly using the standard W-lamp source and LED. System calibration is done by Rayleigh 
scattering for stray light measurements at various pressures by filling the vessel with $\mathrm{N}_{2}$.

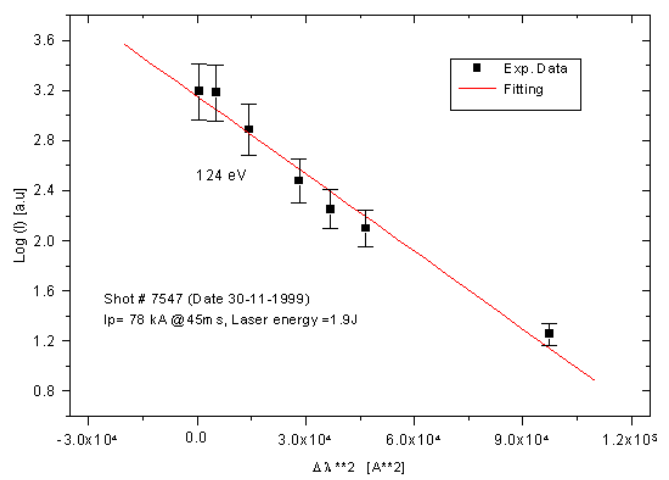

Figure 10. Electron temperature measurement during a typical plasma shot.

\section{Spectroscopy}

The Plasma is monitored with a Grazing Incidence Monochromator (GIM, 10-600nm)) , a Normal incidence spectrometer (NIM, $100-300 \mathrm{~nm}$ ) and a Visible spectrometer(VIS, 300-800nm). Also there are a number of interference filter based spectral line monitors for tracking the spectral lines from low ionization states of Oxygen and Carbon. When fitted with an ICCD Camera at the exit plane, the VIS and NIM can capture a $25.0 / 12.5 \mathrm{~nm}$ wide spectral region centered around any wavelength at intervals of about 15 milliseconds.

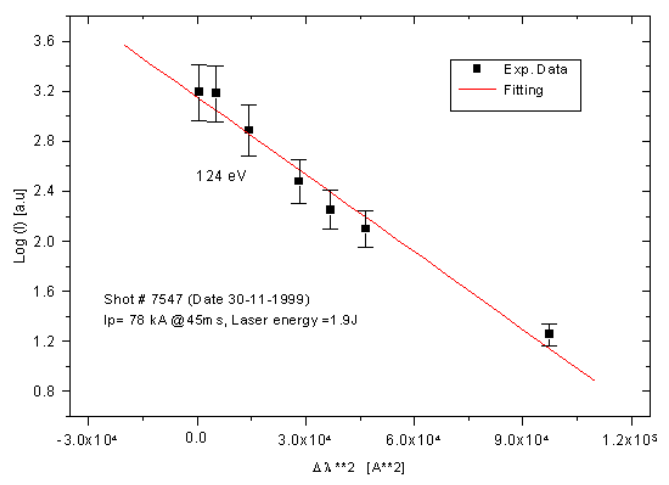

Figure 11. VUV spectra from ADITYA plasma.
The two VUV spectrometers usually view the midplane radial chord but by the use of grazing incidence mirrors, the line of sight can be shifted towards the edges also. They are often used as line monitors whereby a single line is monitored continuously with a time resolution of ) 0.1 millisec.

In the visible wavelength region, a number of $1 \mathrm{~mm}$ core fused silica fibers are used to gather light from different regions of interest in the plasma facing components (in board and outboard limiters, wall etc). For gathering sufficient light from the weak Bremsstahlung radiation along a central chord, a $5 \mathrm{~mm}$ dia 'liquid filled light guide' is set up leading to a PMT detector equipped with a narrowband (1 nm) interference light filter. All the visible wavelength diagnostics are calibrated for absolute intensity measurements.

In the ADITYA discharges of current $\geq 60 \mathrm{KA}$ and duration $\geq 60$ millisec, the absolute intensities of $\mathrm{H}$ and CII, CIII, OII, OIII have been measured and therefrom, the fluxes of $\mathrm{H}, \mathrm{O}$ and $\mathrm{C}$ from the $\mathrm{SS}$ wall and the Graphite limiters been quantified. From the visible BL measurement the Zeff in such discharges has been estimated. The rather high value (Zeff $>6$ ) suggests the possibility of medium $\mathrm{Z}$ impurities.

Presence of Fe, Cr, Si in their low ionization states have been detected in the VUV spectra from NIM, but their concentration is yet to be assessed. The VUV spectra also shows highly ionized states of Oxygen and Carbon (e.g. OVII and CV).

\section{ECE Diagnostics}

Electron Cyclotron Emission (ECE) diagnostic provides very useful measurements of electron temperature and its radial profile. An eight channel E-Band Super Heterodyne Radiometer (SHR) is used to measure ECE on ADITYA tokamak. The temporal and the spatial resolutions of the SHR are $\sim 10 \mu$ s and $\sim 4 \mathrm{~mm}$ respectively. Measurements have been done with different toroidal magnetic fields ranging from $0.75 \mathrm{~T}$ to 1 $\mathrm{T}$. Initially only the optically thin, third harmonic was measured with toroidal magnetic field $0.75 \mathrm{~T}$. Typical signal for such condition on all the eight channels are shown in Fig. 12. 

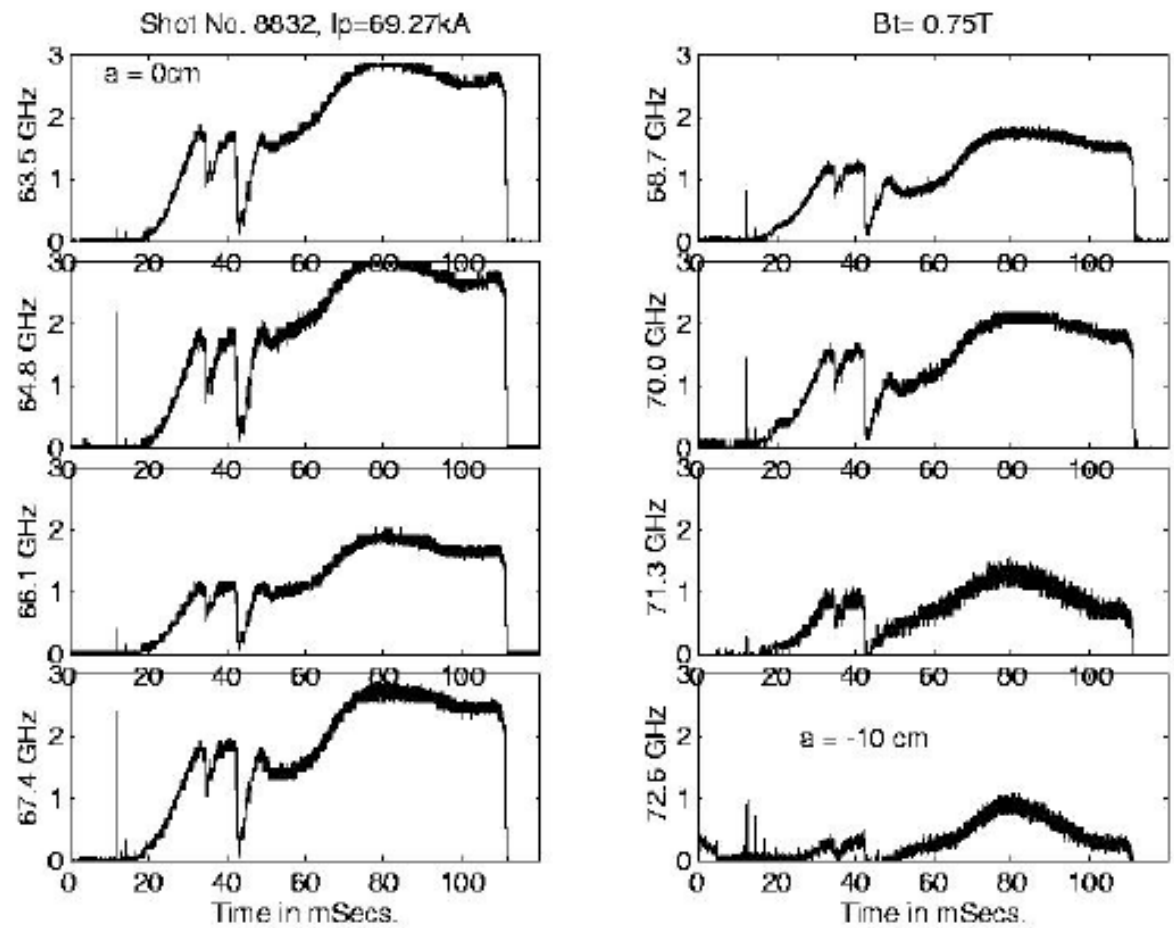

Figure 12. Eight channel ECE radiometer signal for Toroidal field of $0.75 \mathrm{~T}$.

With the help of density measurement by interferometer and reflectivity estimation from the measurement of temperature with soft x-ray diagnostic, efforts are being made to find out the radial profile of electron temperature.

\section{Experimental results}

Edge plasma fluctuation studies are being continued. Array of Langmuir probes are being deployed to measure the fluctuations. This turbulent fluctuation is decomposed into wavelets of different scales. The crossfield transport of fluctuation amplitude is modeled by a nonlinear wavelet coupling equation:

$\mathrm{Y}(\mathrm{k})=\mathrm{L}(\mathrm{k}) \mathrm{X}(\mathrm{k})+\sum_{i, j} \mathrm{Q}(\mathrm{i}, \mathrm{j}) \mathrm{X}(\mathrm{i}) \mathrm{X}(\mathrm{j})$

where, $\mathrm{X}$ and $\mathrm{Y}$ are wavelet transforms of input and output signals; i, j and $\mathrm{k}$ represent wavelet scales $\left(a_{i}=2^{i-1}\right.$ etc. $)$, and $\mathrm{L}(\mathrm{k})$ and $\mathrm{Q}(\mathrm{i}, \mathrm{j})$ are linear and quadratic coupling coefficients respectively. A formalism has been developed for estimating the coupling coefficients. In this formalism, the number of interacting wavelet is small, reducing the degrees of freedom drastically. The amount of data required for the es- timation is significantly smaller than that required in Fourier based formalism. The power transfer due to linear, mixed and quadratic couplings is represented by the respective coherencies.

The estimates of coupling coefficients and coherencies for potential fluctuations in the edge plasma of ADITYA tokamak reveal interesting features. It is observed that linear coupling coefficient and total coherency are close to 1 when the probe separation is small. They fall with increasing probe separation. The characteristic scale lengths vary from $20 \mathrm{~mm}$ at a large scale $(3.5 \mathrm{kHz})$ to $10 \mathrm{~mm}$ at a small scale $(225 \mathrm{kHz})$. The scale length can be interpreted as a cross-field correlation length as determined by the linear coupling. The total coherency is dominated by the linear coherency. The quadratic and mixed coherencies are comparatively small but significant at small scales. The quadratic coupling is not widely spread and is strong only at a few discrete scales. The sign of the power transfer due to mixed coupling is negative. 

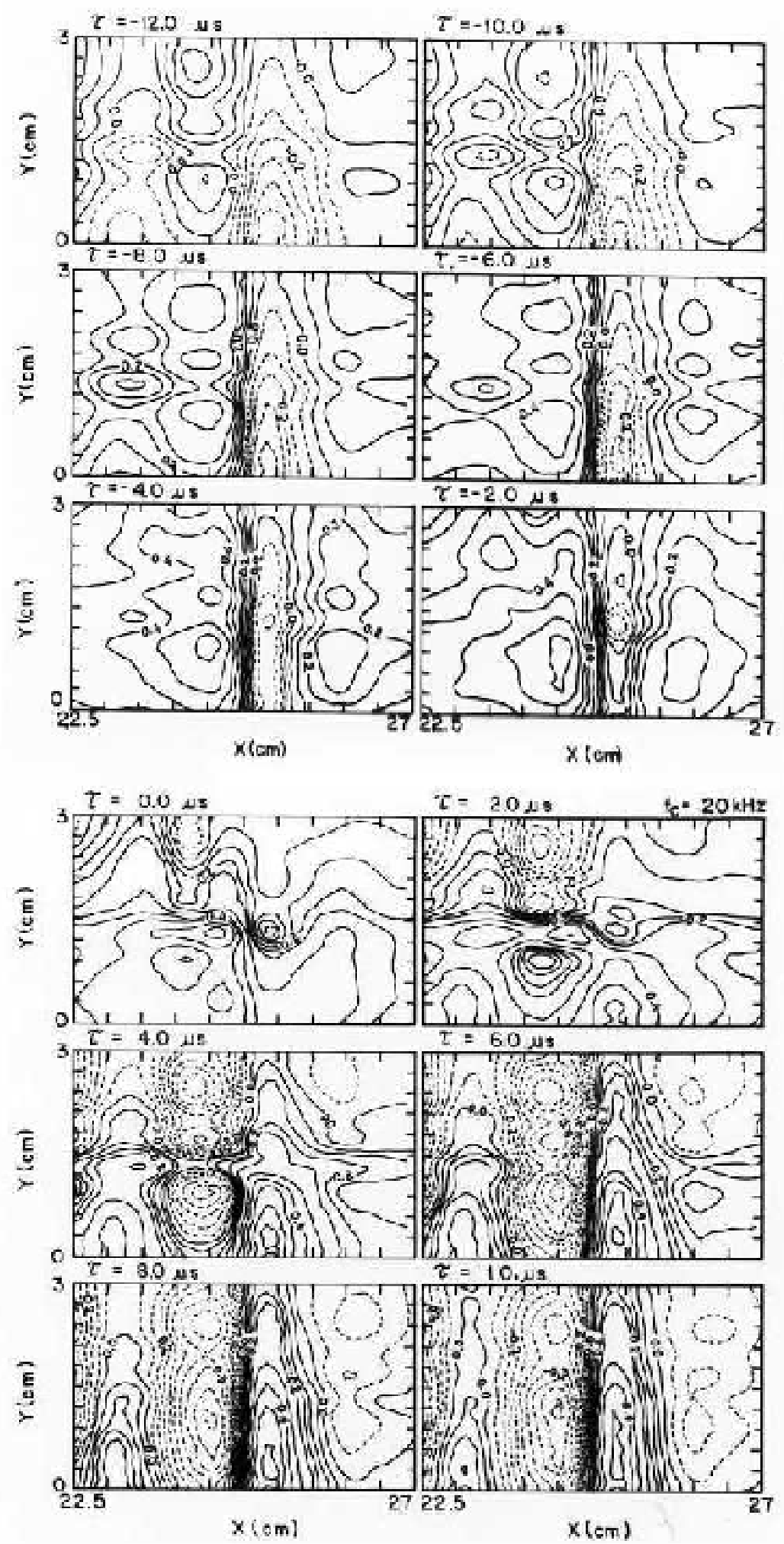

Figure 13. Contours of conditional potential structures when $\Phi_{C}=1.5 \sigma, \mathrm{d} \Phi_{C} / \mathrm{dt}>0$ conditions have been applied on the reference probe. 
Conditional potential structures have been measured in the edge plasma of ADITYA tokamak [9]. The conditional sampling technique allows determination of the potential structure over a section of the poloidal plane from measurements using a radially movable (poloidal) array of Langmuir probes. Fig.13 shows the contours of conditional potential structures when $\Phi_{c}$ $=1.5 \sigma, \mathrm{d} \Phi_{c} / \mathrm{dt}>0$ conditions have been imposed on the reference probe. It is observed that structures are elongated in poloidal direction and seperated on two sides of the last closed flux surface. The radial isolation is broken when the amplitude of the structure is large. The dynamical behaviour of the structure suggests the mechanism of 'bursty' transport of plasma particles in the edge.

The spatial and temporal structures of magnetic signal in the tokamak ADITYA is analysed using recently developed Singular Value Decomposition (SVD) technique. The analysis technique is first tested with simulated data and then applied to ADITYA Mirnov coil data to determine the structure of current perturbation as the discharge progresses. It is observed that during the current rise phase, current perturbation undergoes transition from $\mathrm{m}=5$ poloidal mode structure to $\mathrm{m}=4$ and then to $\mathrm{m}=3$ mode structure. At the time of current termination, $\mathrm{m}=2$ mode structure is observed.

The understanding of fluctuation driven anomalous transport of particle and heat is still of paramount interest in modern fusion devices. The apparent lack of any characteristic time and length scales in the edge plasma fluctuations has prompted a search for scale invariant properties. To that end, the floating potential fluctuations in the Scrape-off layer plasma of ohmically heated ADITYA tokamak have been analyzed. It is observed that the probability distribution function of a sum of $n$ random fluctuations converge to a Levy distribution for $n<40$ whereas for larger $n$, the distribution converges to a Gaussian. The Levy and the Gaussian processes are paradigms of super diffusive and diffusive transport processes respectively. Thus our observation indicates that the transport of small scale fluctuations takes place by convection, whereas large scales follow the diffusive law.

\section{Prediction of major disruption}

A neural network (NN) technique has been used to predict disruptions in tokamak ADITYA. A time series prediction method has been employed whereby a series of past values of some time dependent quantity is used to predict its value in the future. The time varying observables used in the work have different diagnostic signals from 4 Mirnov probes, one soft X-ray monitor and one hydrogen-alpha monitor. The predicted quantities are the same observables at some future time. The neural network has been trained with the past values of the different diagnostic signals as inputs and the future values of the same quantities as targets. The trained neural network is used to forecast in a multistep sequence. This amounts to a prediction several time steps earlier. Very good prediction results are obtained upto $8 \mathrm{~ms}$ earlier with little distortion of the signals and no appreciable time lag, a capability which is believed to be well suited to the task of an on-line prediction of disruption in ADITYA.

As actual experimental signals are used, confidence regarding the performance of the neural network on hardware implementation is automatically ensured.

\section{Prediction of density limit}

An attempt has been made to make a prediction of the disruption boundaries for the density limit disruption case using a NN. Using experimental signals as input, the network should, in the long run, be able to provide information to the real time control systems about the density limit at which the discharge is llikely to disrupt, so that the density can be kept below that limit. Several diagnostic signals are used from the ADITYA tokamak and are presented at selected time instants to the neural network inputs in order to predict, at each of these instants, the density boundary. A disruption threshold has been established in order to examine the possibility of using the network as a real time disruption alarm. For most of the discharges this threshold is reached much before the actual disruption. The NN is also used to make an optimization of the particular set of diagnostics in order to obtain the ones most crucial for predicting the density limit.

\section{Influence of eddy-current}

Influences of eddy currents on the magnetic measurements and the identification process of plasma control parameters using those measurements, as also the optimisation of the magnetic diagnostics have been analysed. This has been conducted with reference to the SST-1 tokamak. Earlier neural network has been used on SST-1 where eddies were not included. To the test data set of that study, about 150 additional data are now added, which included the eddies. It has been found that the poloidal and toroidal beta and the plasma internal inductance are the worst affected by this. The extent to which the different sensors are screened due to the eddy currents have also been calculated for different distributions. Sensor ranking has been made with respect to the degrees of screening and 
compared with the case without eddies.

Results have shown that some of the Mirnov probes within the outboard limiter have been the least affected, while some others have shown strong redundancy effects. One Mirnov probe within the plasma facing side of the inboard limiter has been found to gain importance with the eddies. The lack of redundancy for sensors in the remote areas of the vacuum vessel has again been strongly exhibited, as some of them have been ranked as very important for all the distributions in spite of a reasonably large shielding. Finally, a common feature for the distributions has been that a shielding of less than $5 \%$ generally kept the ranking unaffected.

\section{Auxiliary heating on aditya Ion cyclotron resonance heating (ICRH) system}

Auxiliary heating at ion cyclotron frequency range has been planned and with this in mind a $20-40 \mathrm{MHz}$, $200 \mathrm{KW}$ system has been integrated and commissioned. Flexibility in frequency and minimum power have been arrived at from the existing ADITYA parameters such as ambient toroidal field, energy confinement time during ohmic heating etc.

The complete system has been fabricated in house including the RF generator. RF generator is made up of four stage amplifiers. The last stage is a $200 \mathrm{KW}$ amplifier fed from the earlier $20 \mathrm{KW}$ stage amplifier. The final stage provides feed back loops to monitor, control and limit various output characteristics, operating voltages and current. $50 \mathrm{mt} 6 " / 9 "$ transmission line transmits the power to the experimental hall. It consists of DC isolation, UHV interface and line monitoring systems. The interface between the transmission line and the vacuum vessel of ADITYA consists of movable rf bellow, high power rf feedthrough and is evacuated independently from the tokamak vacuum system. Fast wave antenna is firmly held by it and allows it radial movement of $5 \mathrm{~cm}$. The complete operation is controlled and monitored with the help of a data acquisition and control system. It operates independently during tests and acts with a master trigger from the ADITYA control system during operation.

Before integration of the ICRH system on Aditya, all the subsystems namely RF generator in the new RF Laboratory, layout of the 6" transmission line above the corridor ( $\sim 50 \mathrm{mts})$ to transmit the power to Aditya hall, vacuum interface connecting the transmitting line to the radial port of the tokamak, installation of high power matching stubs with other components in the tokamak hall, integration of the interface structure and UHV pumping system, antenna for fast wave launching inside the tokamak at its preassigned place radially behind the poloidal limiter, diagnostics associated with the rf and edge plasma measurements have been individually tested. After commissioning the system, test runs at low power levels have been conducted to match the system first without plasma and then finally with plasma at different frequencies. Antenna conditioning is done in UHV and in presence of plasma before power is delivered into the plasma.

$200 \mathrm{KW}$ final stage is being tested on the dummy load. Wire burn tests to limit energy deposited in the tube has been performed successfully. $20 \mathrm{KW}$ rf power is applied to the fast wave antenna positioned $0.5 \mathrm{~cm}$ behind the movable limiter. Antenna is conditioned by applying train of short pulses.

ICRF breakdown of initial plasma and wall conditioning in a steady state tokamak would be prefered to the conventional schemes due to the fact that the same heating system could be used at full toroidal magnetic field between successive shots. Therefore, besides heating, the same system is being used to experiment on plasma breakdown and preionization to help ohmic break down. Keeping in view the need for such a scheme on SST-1, experiments are underway to study plasma breakdown at different values of toroidal magnetic field at different pressures. RF wall conditioning would start soon.

\section{Electron cyclotron resonance frequency (ECRF) system}

The paramerter space of operation of ADITYA is affected by the stray magnetic fields. Electron cyclotron resonance breakdown for preionization and startup has been incorporated recently on ADITYA. For this purpose, an Electron Cyclotron Resonance Heating (ECRH) system has been installed and commissioned for operation on tokamak Aditya. The system would serve dual purpose of electron heating during a normal discharge and initial breakdown to help increase the operational window. The $200 \mathrm{KW}$ gyrotron, VGA$2000 \mathrm{~A} 19$, operating at a frequency of $28 \pm 0.1 \mathrm{GHz}$ is first commissioned into a dummy load for pulsed operation. The output power in TE-02 mode is measured by various techniques. Paper burn pattern is measured at the tube output to confirm the mode structure. 


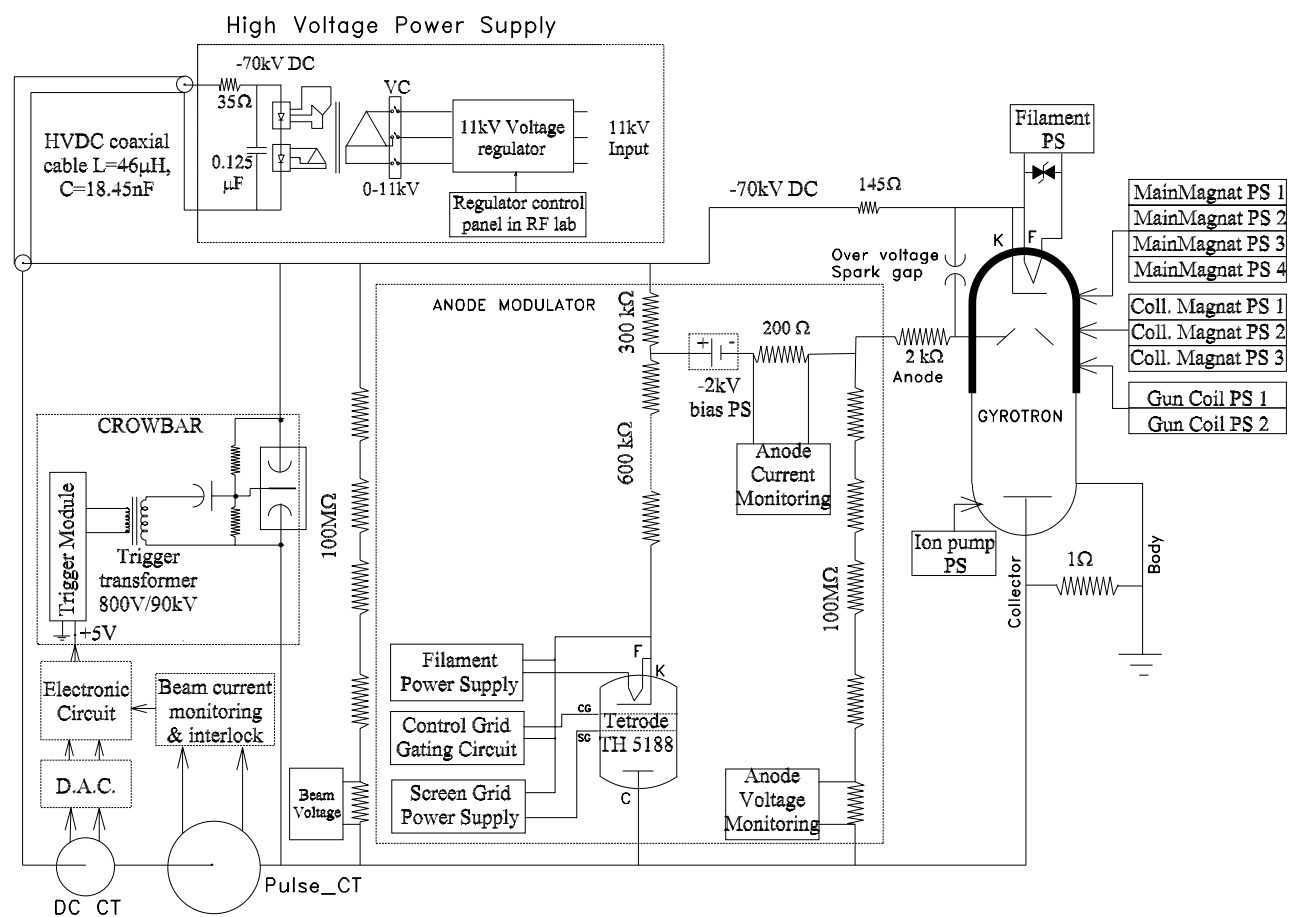

Figure 14. Schematic of the Gyrotron testing scheme showing various electrical requirements.

A Matching Optics Unit is used to convert the TE02 mode to a HE-11 mode which is then transmitted through a $\sim 10$ meter long transmission line. The total attenuation of the transmission line is measured to be less than 1.1dB. Paper burn patterns at various locations of the transmission line confirm the mode structure. The schematic of the gyrotron testing is shown in Fig.14. The gyrotron operation is remotely controlled using the data acquisition and control (DAC) system.

The system has been commissioned in two stages. First the gyrotron output power has been measured in a dummy load. Then the out put power is converted to HE11 mode before transmitting through the line components, vacuum window to the BLS.

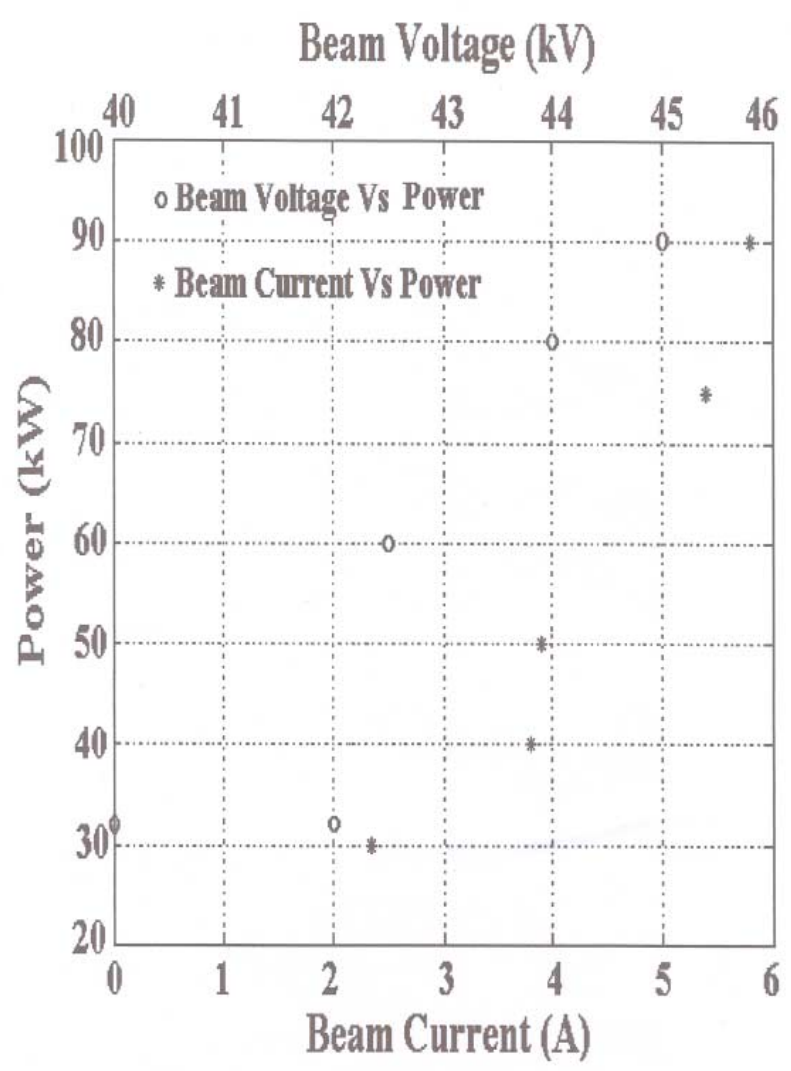

Figure 15. Microwave output power as a function of beam voltage and beam current (at $-42 \mathrm{kVDC}$ of Beam Voltage). 
Initially the tube is conditioned by applying high voltage for short durations. Gradually, the pulse length is increased, the beam voltage is raised to $-50 \mathrm{kV}$ and the ion pump current is not allowed to exceed $20 \mu \mathrm{A}$ during this process.

Fig.15 shows the output power as a function of beam voltage and beam current. Due to stiffness of the potential divider anode voltage did not increase beyond $23 \mathrm{kV}$ and hence operation is restricted to $-48 \mathrm{kV}$ beam voltage with pulse width slowly increased to $300 \mathrm{msec}$ due to restriction of water flow in the dummy load to $45 \mathrm{lpm}$.

The maximum measured output power for a period of $300 \mathrm{~ms}$ pulse length, is $\sim 90 \mathrm{KW}$ at a cathode voltage of $-48 \mathrm{kV}$ and $\sim 6.5 \mathrm{~A}$ beam current. Burn pattern of the microwave output power confirms the factory result of a $93 \%$ pure $\mathrm{TE}_{02}$ mode.
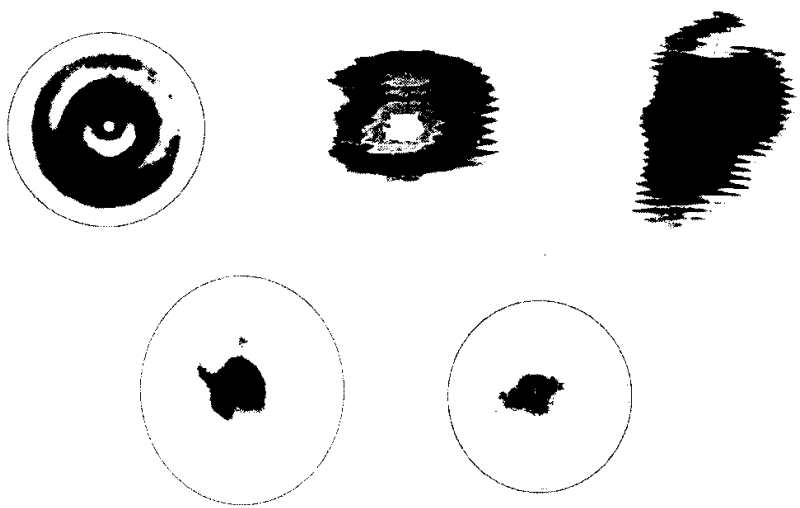

Figure 16. Burn patterns at various locations : i) gyrotron output, ii) 1st mirror of MOU, iii) 2nd mirror of MOU, iv) polariser output, v) transmission line output.

Then the HE-11 gaussian beam is transmitted through the over moded corrugated wave guide, mitre bend, polariser and a boron nitride barrier window to fall on the beam launching system.

Fig.16 shows the burn pattern at different locations of the transmission line.

The Beam Launching System (BLS) consists of two matellic mirrors to steer and focus the beam at centre of the vessel to initiate the breakdown.. BLS has been designed and fabricated indigenously. It has been coupled to Aditya vacuum system and the vacuum compatibility has been established before microwave power is introduced.

The gaussian beam entering the BLS is focussed at the minor axis. The tokamak gas feed controls the background pressure. Spectroscopic diagnostics and Langmuir Probes positioned at plasma edge measures various quantities during breakdown of the gas. The pressure and the magnetic field are varied to study the breakdown plasma.

Fig. 17 shows the data collected during breakdown phase of the plasma. A plasma density of $\sim 5 \times 10^{11}$ per cc is obtained. Thus, during this preliminary experiment, it is concluded that with the available HVDC power supply system, $300 \mathrm{msec}$ operation of the gyrotron at $100 \mathrm{~kW}$ of output power has been obtained successfully. The power is then transmitted through the transmitted line and deposited at ADITYA minor axis to obtain successful plasma breakdown.

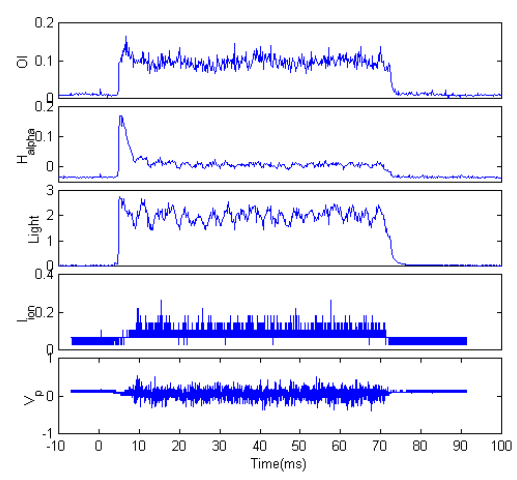

Figure 17. Breakdown data for $\mathrm{O}_{2}$ line, $\mathrm{H}_{\alpha}$, visible total light, plasma density $\left(\mathrm{n}_{e}\right)$ and floating potential $(\Phi)$ at a pressure $4 \times 10^{-5}$ torr.

\section{Summary}

Most of the subsystems for SST-1 have been engineered and are under fabrication. Winding of most of the SC coils and copper coils are over. The casings for TF coils are being fabricated. A fabrication trial of the prototype one sector vacuum vessel and cryostat has been successfully completed and commissioned at site. In the meantime fabrication of vaccum vessel and cryostat have started. The design of PFCs have been completed. Structures are being fabricated. Graphite slabs are being procured which will then be machined as per requirement and conditioned subsequently. A vacuum furnace has been commissioned for this purpose. Fabrication of RF systems is in advanced state. In the meantime $2 \times 500 \mathrm{KW} \mathrm{CW}$ Klystrons at $3.7 \mathrm{GHz}$ have been successfully commissioned on dummy loads for $1000 \mathrm{Sec}$. All prototype components have been high power tested. Support structures, vacuum window, grill structure are being fabricated. In the ICRH system, $1.5 \mathrm{MW}$ final stage of the generator is being fabricated. Driver stage at $200 \mathrm{KW}$ is being tested on the $1.5 \mathrm{MW}$ dummy load. Gyrotron and the transmission line for 
the ECRH system at $82.6 \mathrm{GHz}$ is being procured. Beam launching systems for ECRH system are being fabricated.

Many expensive components and instruments of various diagnostics have been procured and tested. Other components are being fabricated. The refrigeration system for $\mathrm{SC}$ magnets is being erected. Commissioning phase would be initiated soon. System engineering and hardware procurement for the data acquisition and control system is in progress. Erection of various systems at site would commence by the end of 2001 . This phase including commissioning and integration of required subsystems to initiate SST - 1 operation would consume more than a year.

Many new experiments are being planned in the next campaign on ADITYA. Detail experiments on RF breakdown and startup with the help of ICRH system and the ECRH system would be conducted. Edge turbulence work would be concluded soon. Plasma discharges would be devoted to install some of the diagnostics. Boronization and lithiumization experiments would be conducted to finalise the process to be employed on SST-1. Finally auxiliary heating with the help of ICRH and ECRH to design values would form a part of experimentation.

\section{References}

[1] Y.C. Saxena, SST-1 Team, Present Status of the SST-1 project, Nucl. Fusion, 40, 1069 (2000).

[2] S.B. Bhatt et al., ADITYA- The First Indian Tokamak, Ind. J. Pure Appl. Phys. 27, 710 (1989).

[3] S. Pradhan, et al., Superconducting Cable-in-Conduit Conductor for SST-1 magnets, Rep. 99-06(67), 2, Kyushu Univ., Furuku, 482, 1999

[4] D. Chenna Reddy, + et al., Design of Plasma Facing Components of SST-1, Rep. 99-06(67) Vol. III, Kyushu Univ., Furuku, 572, 1999

[5] D. Bora and RF Group, RF Systems on SST-1 Tokamak, 20th Symposium on Fusion technology, 1998

[6] A.K. Chakraborty, et al., in Fusion Technology (Proc. 19th Symp. Lisbon, 1996), Elsevier, Amsterdam and New York, 657, 1996

[7] D. Bora, et. al., High power continuous wave microwave system at 3.7 GHz, Rev. Sci. Instru., 72, 1869 (2001).

[8] P. Ranjan, et. al., Central control system for SST 1 Tokamak, Rep 99 - 06(67), vol III, Kyushu Univ., Furuku, 722, 1999

[9] B.K. Joseph, R. Jha, et. al., Observation of vortex-like coherent structuress in the edge plasma of the ADITYA tokamak, Phys. Plasmas, 4, 4292 (1997). 